\title{
Die „Exchange-Veranstaltungsreihe“ - ein Planungs- und Steuerungsinstrument für transdisziplinäre Forschungsprojekte
}

Christina Benighaus, Belen Zevallos, Charlotte Eller, Martina Hilligardt, Mandana Alimardani \& Ludger Benighaus

\section{Einleitung und Kontext}

Kern eines jeden Reallabors ist die Zusammenarbeit von Akteuren aus der Wissenschaft (Forscher(innen)) mit Akteuren aus der für das Projekt relevanten Praxis, um gemeinsam Wissen zu erzeugen (Forschungsziel), Transformationsprozesse $\mathrm{zu}$ fördern und $\mathrm{zu}$ gestalten (Praxisziel) und individuelle und gesellschaftliche Lernprozesse anzustoßen (Bildungsziel) (Beecroft et al. 2018, S. 79ff.). Reallabore bedürfen daher geeigneter Formate für die Zusammenarbeit verschiedenster Akteure (Schäpke et al. 2017; Arnold und Piontek 2018). Dies gilt analog ebenso für andere transdisziplinäre (und transformative) Forschungsformate und -projekte. Die in diesem Beitrag erläuterte Planung einer Veranstaltungsreihe ist ein Werkzeug zur detaillierten Gliederung und Steuerung eines transdisziplinären Forschungsprojekts, bei dem die transdisziplinäre Arbeit maßgeblich mit externen Beteiligten erfolgt, die punktuell in die Forschungsarbeit involviert werden (s. dazu Defila et al. 2006, S. 214ff.). Es führt zu mehreren transdisziplinären Veranstaltungen, die in Form von öffentlichen Symposien mit jeweils anschließendem projektinternen Reflexionsworkshop durchgeführt werden. Mithilfe jeder dieser Veranstaltungen wird ein abgegrenzter Ort der transdisziplinären Wissensproduktion zur Verfügung gestellt. Die Verknüpfung mehrerer solcher Veranstaltungen zu einer Veranstaltungsreihe - der „Exchange-Veranstaltungsreihe" - gibt die Struktur für die Zusammenarbeit zwischen Akteuren aus der Wissenschaft und Akteuren aus der Praxis im Forschungsprojekt vor. Sie ermöglicht es, die gesamte Projektplanung entlang der Veranstaltungsreihe zu organisieren, zu steuern sowie eine aktive Schnittstelle zwischen Wissenschaft und Praxis zu schaffen.

Die Planung der Exchange-Veranstaltungsreihe geht in Anlehnung an Eckart et al. (2018, S. 127) von vier Phasen eines Forschungsprozesses aus: Einstiegsphase, Orientierungsphase, Arbeitsphase und Abschlussphase. Diese spiegeln sich 
in den einzelnen Veranstaltungen der Reihe wider. Die Veranstaltungen dienen für die jeweilige Phase als Meilensteine und wirken dadurch Struktur gebend sowohl für die einzig an den Veranstaltungen beteiligten Akteure als auch für das Projektteam selbst. Gleichzeitig dienen sie auf inhaltlicher Ebene der Verknüpfung der verschiedenen Erkenntnisse - beispielsweise aus mehreren Teilprojekten - im Forschungsprojekt, indem diese Erkenntnisse aus den einzelnen Teilen des Projekts im Zuge der Veranstaltungen gemeinsam reflektiert und angereichert werden. ${ }^{1}$ Die Exchange-Veranstaltungsreihe stellt ein Instrument dar, durch welches externe Akteure insbesondere aus der Praxis (Anwender(innen), Nutzer(innen) im Themenbereich des Projekts), aber auch aus der Wissenschaft (für das Projekt relevante Forscher(innen)) punktuell im Zuge der Projektlaufzeit beteiligt werden. Als ,externe Akteure' werden hier jene Akteure aus dem ,äußeren Akteurskreis“ im Sinne von Seebacher et al. (2018, S. 157) bezeichnet, die nicht aktiv im Forschungsprojekt eingebunden sind (also nicht Teil des Projektteams sind, weder als Praxispartner noch als Forscher(innen)), sondern sich in der jeweiligen Veranstaltung entsprechend einbringen. Das im Projekt generierte Wissen wird im Rahmen der Veranstaltungen einem größeren Kreis an Akteuren präsentiert, gemeinsam mit diesen externen Akteuren reflektiert und durch die gemeinsame Wissensproduktion von Projektteam, externen Forscher(inne)n und Praxisakteuren erweitert. Durch die Verknüpfung der Veranstaltungen wird gleichzeitig eine Lernumgebung geschaffen, die den Beteiligten einen kontinuierlichen, geschützten Rahmen für Information, Austausch, Kooperation sowie Evaluation und Reflexion bietet (Beecroft et al. 2018, S. 83).

Das in diesem Beitrag beschriebene Werkzeug wurde im Rahmen des Reallabors „STADT-RAUM-BILDUNG - Reallabor für die nachhaltige Planung von Bildungslandschaften und die Integration von Aus- und Umbauten von Schulgebäuden“ (Reallabor SRB, s. auch Steckbrief im Anhang zu diesem Buch) entwickelt. Im Reallabor SRB standen Sekundarschulen in Baden-Württemberg im Fokus, die aktuellen Umwälzungen auf politischer Ebene sowie notwendigen strukturellen und baulichen Veränderungen ausgesetzt waren. Die hohe gesellschaftliche und politische Aktualität veranlasste das Projektteam, bereits im Antrag mehrere öffentliche Symposien in regelmäßigem zeitlichem Abstand vorzusehen. Da Bildungsthemen in Baden-Württemberg auf (politischer) Landesebene entschieden werden, sollte ein möglichst breiter Kreis an Akteuren aus dem ganzen Bundes-

1 Im Beitrag wird die gängige Projektstruktur zugrunde gelegt, dass inter- und transdisziplinäre Forschungsprojekte aus mehreren Teilen bestehen, die auf gemeinsame Ziele und Ergebnisse ausgerichtet sind. In diesen Teilen, die hier als ,Teilprojekte' bezeichnet werden (und die selbst disziplinär, interdisziplinär oder transdisziplinär sein können), finden „Forschungsarbeiten statt, die zu planen und aufeinander abzustimmen sind, die aber gleichzeitig einen eigenen Stellenwert haben und auch eigenständige Ergebnisse hervorbringen" (Defila et al. 2006, S. 38). 
land und darüber hinaus beteiligt werden. Hierzu wurden gezielt Personen eingeladen, die sich kurz vor oder mitten in einem Veränderungsprozess einer Schule befanden. Im Fokus standen zunächst Schlüsselpersonen, die bei diesen Veränderungen an Schulen eine wesentliche Rolle einnahmen. Zu diesen Personen gehörten Vertreter(innen) der kommunalen Verwaltungen, Schulleitungen und Politiker(innen) vorwiegend aus den Stadt- und Gemeinderäten. Des Weiteren wurden Personen aus den Kollegien einzelner Schulen, Architekt(inn)en verschiedener Büros sowie Wissenschaftler(innen) aus thematisch verwandten Forschungsprojekten adressiert, so dass eine breite Mischung verschiedener Akteure mit unterschiedlichen Wissensbeständen und Interessen entstand (s. hierzu Benighaus und Renn 2016, S. 70ff.). Durch landesweite Einladungen wurden nicht nur verschiedene Akteursgruppen, sondern auch Personen der gleichen Akteursgruppe, jedoch aus unterschiedlichen Kontexten, zusammengebracht. Somit konnten sich beispielsweise Vertreter(innen) verschiedener Kommunen über ihre jeweiligen individuellen Herausforderungen austauschen. Das wiederholte Zusammentreffen der teilnehmenden Personen führte zu einem zunehmenden Vertrauensverhältnis und einer Zusammenarbeit zwischen den Teilnehmer(inne)n, die zum Teil auch jenseits der vom Reallabor SRB organisierten Veranstaltungen weitergeführt wurde.

Das im Folgenden beschriebene Werkzeug der Planung einer Veranstaltungsreihe eignet sich - abgeleitet aus den Erfahrungen des Reallabors SRB - bei gesellschaftlich aktuellen und politisch kontrovers diskutierten sowie in den aktuellen Medien vielfach besprochenen Themen. Die aus diesen Rahmenbedingungen entstehende hohe Dynamik im Feld - sowohl hinsichtlich neuer Erfahrungen und Praktiken als auch hinsichtlich der sich verändernden Akteurslandschaft - kann $\mathrm{zu}$ einer chancenreichen, aber auch zu einer konfliktbehafteten Zusammenarbeit zwischen den Akteuren sowie zwischen dem Forschungsprojekt und den Akteuren im Feld, die nicht am Projekt beteiligt sind, führen. Dieser Herausforderung kann durch eine Veranstaltungsreihe Rechnung getragen werden, da die jeweils aktuellen Akteure mit ihrem jeweils aktuellen Wissen wiederholt über die gesamte Projektlaufzeit hinweg einbezogen werden. Dies führt bezogen auf die Forschung im Projekt zu Iterationsschleifen im Prozess, so dass das jeweils neue Praxiswissen immer wieder reflektiert und in die Forschung integriert werden kann und so die Forschungsergebnisse mit der Dynamik im Feld Schritt halten können.

Bei einem Forschungsthema, das die vorher beschriebenen Merkmale erfüllt, kann es zudem wichtig sein, dass sich das Forschungsprojekt als Akteur im Feld präsentiert und legitimiert, um das Interesse der Akteure am Projekt und an der gemeinsamen Arbeit zu wecken und zu halten (Quint et al. 2018, S. 71). Zudem eignet sich das Instrument einer Veranstaltungsreihe speziell für Forschungsprojekte, die so angelegt sind, dass sie sich nicht nur auf einen Ort beziehen, 
sondern ein Thema bearbeiten, das mehrere Orte oder eine ganze Region betrachtet. Gerade wenn der Fokus der Forschung eher auf einer allgemeinen Problemstellung und weniger auf der lokalen Verortung des Problems liegt, ist das Instrument besonders geeignet. Denn um eine Vielfalt an Erfahrung und Wissen aus Wissenschaft und Praxis zu integrieren, können durch eine Exchange-Veranstaltungsreihe externe Forscher(innen) und Praxisakteure aus verschiedenen Kontexten punktuell integriert werden (s. zur Einbindung von Akteuren in Reallaboren Alcántara et al. 2018, S. 138ff.). Dadurch kann die notwendige Bandbreite des Wissens für das Projekt fruchtbar gemacht werden.

Einen besonderen Nutzen bietet der vorliegende Beitrag für alle, die sich in der Planung eines transdisziplinären Forschungsprojekts befinden. Diese Planung wird meist von denjenigen Wissenschaftler(inne)n übernommen, die auch nach Genehmigung des Projekts und Start der Forschung zum „Kernbereich“ gehören werden, also Teil des für die „Konzeption, inhaltliche Kohärenz, Prozessgestaltung und organisatorische Steuerung verantwortlichen Teams" sein werden (Seebacher et al. 2018, S. 157). Der Beitrag stellt eine Anleitung zur Planung einer transdisziplinären Veranstaltungsreihe als ,Gerüst ${ }^{`}$ des transdisziplinären Projektdesigns dar. Die Exchange-Veranstaltungsreihe wird im Folgenden an einem allgemeinen Projektablauf vorgestellt, der natürlich in jedem transdisziplinären Forschungsprojekt individuell variieren kann, so dass das Instrument entsprechend angepasst werden muss.

Im Folgenden wird die Exchange-Veranstaltungsreihe erläutert, wobei zuerst auf die Planung der Veranstaltungsreihe eingegangen wird (Kapitel 2), bevor die einzelnen Veranstaltungen vertieft dargestellt werden (Kapitel 3). Abschließend werden das Instrument und seine Anwendung im Reallabor SRB noch einmal reflektiert (Kapitel 4). Einzelne Beispiele aus dem Reallabor SRB in Kapitel 3 zeigen die Anwendung in diesem Forschungsprojekt und deren Mehrwert (s. die SRBBoxen).

\section{Die Exchange-Veranstaltungsreihe im Überblick und ihre Planung}

Durch die Exchange-Veranstaltungsreihe wird die Projektlaufzeit in mehrere Veranstaltungen, bestehend aus öffentlichen Symposien mit jeweils angeschlossenem internem Reflexionsworkshop, gegliedert (s. Tab. 1). Ein wichtiges Ziel der Symposien ist es, die Transparenz des Forschungsprojekts zur gewährleisten und einer breiteren Öffentlichkeit die Möglichkeit zu geben, die Inhalte des Forschungsprojekts zu erfassen und zu kommentieren. Die am nächsten Tag stattfindenden, internen Reflexionsworkshops wiederum bieten einen gesteuerten Austausch innerhalb des Projektteams. Dieses Vorgehen führt zu einer klaren Konstituierung 
von ,Innen“ und ,Außen“ (s. dazu Quint et al. 2018, S. 71ff.). Zum ,inneren“ Kreis zählen die aktiv am Forschungsprojekt Beteiligten. Der regelmäßige Austausch in den Reflexionsworkshops und das dort stattfindende Reflektieren des Symposiums vom Vortag fördern das Vertrauensverhältnis im Projektteam und eine konstruktive Zusammenarbeit im weiteren Forschungsverlauf. Zusätzlich führt das gemeinsam Erlebte und Reflektierte der Symposien zu gemeinsamen Erfahrungen des Projektteams und unterstützt dadurch die Konsens- und Synthesebildung (Defila et al. 2006, S. 35-38). Damit dies gelingen kann, wird die Veranstaltungsreihe bereits im Forschungsantrag verankert und bildet die Grundlage des Forschungsdesigns.

Die folgenden Ausführungen erläutern, wie die Veranstaltungsreihe noch vor Beginn der eigentlichen Forschung geplant wird. Dabei sind einzelne Veranstaltungen zwingend, während andere optional sind, d. h. abhängig von der Dauer des Forschungsprojekts und von den gewünschten Schwerpunkten vorgesehen oder weggelassen werden können. Wie die einzelnen Veranstaltungen dieser Reihe also die Symposien mit jeweils anschließendem Reflexionsworkshop - im Speziellen geplant und durchgeführt werden können, wird in Kapitel 3 ausgeführt.

\subsection{Die Exchange-Veranstaltungsreihe im Überblick}

Die Exchange-Veranstaltungsreihe gliedert die gesamte Projektlaufzeit in zeitliche Abschnitte, definiert Meilensteine im Projekt und erlaubt es, die Reihe als ein Steuerungsinstrument zu nutzen. Beispielsweise können so Teilprojekte, aus denen das Forschungsprojekt bestehen kann, sinnvoll miteinander vernetzt werden. Dadurch wird bereits zu Beginn des Forschungsprozesses sichergestellt, dass im Verlauf des Projekts Wissen aus den Teilprojekten zusammengeführt wird, um die Synthesebildung zu unterstützen. Zentral ist dabei, dass sich die beteiligten Akteure ihrer gleichzeitigen Rollen als Expert(inn)en und Laien im Kommunikationsprozess bewusst sind und die Sichtweise des ,fachfremden' Gegenübers annehmen (s. dazu z. B. Godemann 2005, S. 85f.).

Sowohl die einzelnen Symposien als auch die jeweils anschließenden Reflexionsworkshops dienen der interdisziplinären Verknüpfung der Wissensbestände der beteiligten Forscher(innen) und der transdisziplinären Integration von Praxiswissen. Über die Symposien wird das Praxiswissen, aktiviert' und ,abgerufen'. In den Reflexionsworkshops diskutieren die Forscher(innen) die Erkenntnisse aus dem jeweils vorausgegangenen Symposium quer zu den beteiligten Disziplinen, werten sie anschließend wissenschaftlich aus und integrieren sie in die laufende Forschung. Zudem wird der Stand der Arbeit in den einzelnen Teilprojekten innerhalb der Projektteams besprochen, und unter Berücksichtigung der durch die externen Akteure neu gewonnenen Erkenntnisse werden die Fragestellungen und Ziele des Gesamtprojekts und der Teilprojekte überarbeitet. 
Tabelle 1: Die Veranstaltungen, aus denen die Exchange-Veranstaltungsreihe besteht.

\begin{tabular}{|c|c|}
\hline $\begin{array}{l}\text { Veranstaltung 1: } \\
\text { Tür-Öffnerin }\end{array}$ & $\begin{array}{l}\text { Steht zu Beginn des Projektverlaufs in der Einstiegsphase: } \\
\text { Zur Etablierung des Forschungsprojekts und Gewinnung } \\
\text { von (weiteren) Praxisakteuren für die transdisziplinäre } \\
\text { Zusammenarbeit. Das Forschungsprojekt und das Projekt- } \\
\text { team werden vorgestellt, das im Forschungsantrag dar- } \\
\text { gelegte Zielwissen wird durch externe Akteure reflektiert } \\
\text { und ergänzt. }\end{array}$ \\
\hline $\begin{array}{l}\text { Veranstaltung } 2 \\
\text { (optional): } \\
\text { Weg-Bereiterin }\end{array}$ & $\begin{array}{l}\text { Optionale Veranstaltung in der Orientierungsphase zwi- } \\
\text { schen Anfang und Mitte des Projektverlaufs: Den Fokus } \\
\text { bilden die transparente Darstellung des Forschungsgegen- } \\
\text { stands, Berichte der Praxispartner (z. B. aus Fallbeispielen } \\
\text { des Projekts) sowie die Integration von Praxiswissen in } \\
\text { den Forschungsprozess. }\end{array}$ \\
\hline $\begin{array}{l}\text { Veranstaltung 3: } \\
\text { Weg-Begleiterin }\end{array}$ & $\begin{array}{l}\text { Findet circa zur Halbzeit der Projektlaufzeit statt, also } \\
\text { während der Arbeitsphase: Sie dient einer ersten Diskus- } \\
\text { sion der praktischen Anwendbarkeit erster Eindrücke aus } \\
\text { der Forschung und der Rückkopplung der Erfahrungen mit } \\
\text { dem wissenschaftlichen Diskurs. }\end{array}$ \\
\hline $\begin{array}{l}\text { Veranstaltung } 4 \\
\text { (optional): } \\
\text { Ziel-Erspäherin }\end{array}$ & $\begin{array}{l}\text { Steht zwischen Mitte und Ende des Projektverlaufs, also } \\
\text { ebenfalls in der Arbeitsphase: Sie dreht sich um die Vor- } \\
\text { stellung erster Teilergebnisse und die Rückspiegelung der } \\
\text { Erkenntnisse aus der Forschung an die externen Praxis- } \\
\text { akteure sowie um die gemeinsame Erarbeitung von Trans- } \\
\text { formationswissen. }\end{array}$ \\
\hline $\begin{array}{l}\text { Veranstaltung 5: } \\
\text { Ziel-Beschreiterin }\end{array}$ & $\begin{array}{l}\text { Findet am Ende des Projektverlaufs statt, also in der Ab- } \\
\text { schlussphase: Hier geht es um die Darstellung der Ge- } \\
\text { samtergebnisse und um deren Umsetzung. Generiertes } \\
\text { Transformationswissen, das im Projekt entstanden ist, } \\
\text { kann so in die Praxis und in folgende Forschungen inte- } \\
\text { griert werden. }\end{array}$ \\
\hline \multicolumn{2}{|c|}{$\begin{array}{l}\text { Reflexionsworkshop: An jeder der Veranstaltungen folgt nach dem öffentlichen Sym- } \\
\text { posium ein interner Reflexionsworkshop, in dem das Projektteam die Beiträge und } \\
\text { Impulse der externen Praxisakteure und ggf. der externen Forscher(innen) aufbereitet } \\
\text { und auswertet. }\end{array}$} \\
\hline
\end{tabular}

Die Exchange-Veranstaltungsreihe dient somit der regelmäßigen Vernetzung und Integration der Ergebnisse aus den Teilprojekten. Die Veranstaltungen fungieren als ,Verknüpfungspunkte' eines Netzes an verschiedenen Wissensbeständen. Während sich in der Einstiegs- und Orientierungsphase erste gemeinsame Themen für die Synthese konkretisieren, werden die Schnittstellen in der Arbeitsphase zunehmend interessanter. Das konsequente Herstellen von Transparenz be- 
zogen auf Forschungsprozesse der Teilprojekte erhöht diese Dynamik und unterstützt dadurch die Bildung von Querschnittswissen und das Entstehen einer Synthese. Welche Arbeitsschritte zur Umsetzung der, Verknüpfungsfunktion' bei den einzelnen Veranstaltungen notwendig sind, wird in Kapitel 3 unter Aufgabenpaket 6 „Dokumentation und Auswertung“ erläutert.

Der folgende Abschnitt beschreibt, wie die Planung der Exchange-Veranstaltungsreihe und die darauf bezogene Steuerung der Vernetzung von Teilprojekten innerhalb des Forschungsprojekts realisiert werden.

\subsection{Die Planung der Exchange-Veranstaltungsreihe}

Für die Planung der Exchange-Veranstaltungsreihe wird, wie bereits erwähnt, von einer vierstufigen Gliederung eines transdisziplinären Forschungsprojekts ausgegangen: Einstiegsphase, Orientierungsphase, Arbeitsphase und Abschlussphase (Eckart et al. 2018, S. 127), die mithilfe der Veranstaltungsreihe strukturiert werden.

\section{Schritt 1: Erstellung des Zeitplans für die Exchange-Veranstaltungsreihe}

Um den Zeit- und Arbeitsplan festzulegen, muss entschieden werden, aus wie vielen Veranstaltungen die Reihe besteht und welche Projektschritte dadurch definiert werden. Dies kann in jedem Forschungsprojekt variieren. Zur Vorbereitung der jeweils nächsten Veranstaltung hat sich im Reallabor SRB ein Rhythmus von neun Monaten bewährt. Ein kürzerer Rhythmus, beispielsweise von einem halben Jahr, kann zur Überforderung der externen Akteure (vor allem der Praxisakteure) aufgrund deren begrenzter Zeitbudgets führen und erfordert zusätzliche Ressourcen im Projektteam, da in diesem Fall die Aufgaben des Veranstaltungsmanagements und der inhaltlichen Forschungsarbeit personell getrennt werden müssen. Ein längerer Rhythmus als neun Monate kann dagegen eine Reduktion des Interesses der externen Praxisakteure mit sich bringen und bietet gleichzeitig eine zu schmale Kommunikationsbasis für den internen Kreis, was wiederum den Aufbau des Vertrauensverhältnisses für eine gemeinsame konstruktive Arbeit gefährdet. Ausgehend von einem neunmonatigen Rhythmus ergibt sich ein Projektverlauf, der im Folgenden exemplarisch für ein dreijähriges und für ein fünfjähriges Forschungsprojekt dargestellt wird.

Für ein dreijähriges Forschungsprojekt sind drei bis vier Veranstaltungen sinnvoll, je nachdem, wie viel Bedarf für die projektinterne Vor- und Nachbereitung zu Beginn und zum Ende des Forschungsprojekts besteht. Bei drei Veranstaltungen plant man die erste Veranstaltung am Ende der Einstiegsphase (Veranstaltung 1: Tür-Öffnerin, s. Abschnitt 3.1), die zweite in der Arbeitsphase (Veranstaltung 3: Weg-Begleiterin, s. Abschnitt 3.3) und die dritte in der Abschlussphase 


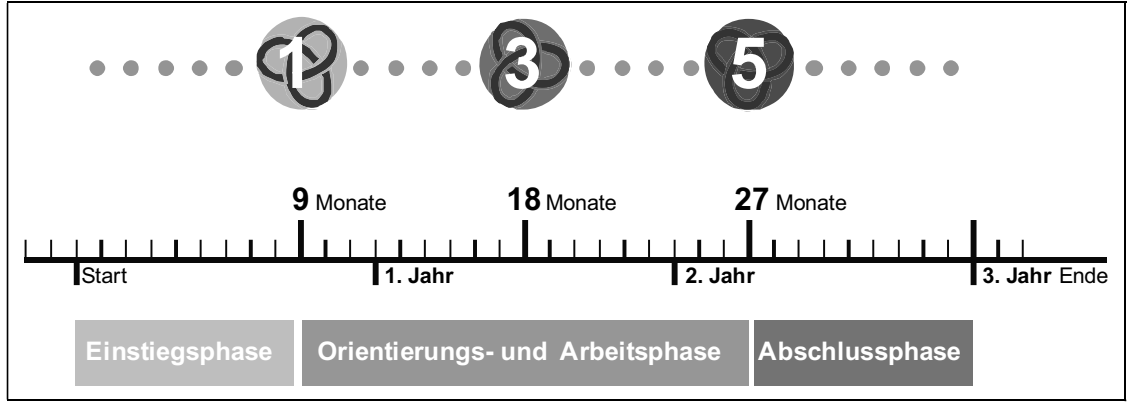

Abbildung 1: Zeitplan eines dreijährigen Forschungsprojekts mit drei Veranstaltungen. (C) Reallabor SRB.

(Veranstaltung 5: Ziel-Beschreiterin, s. Abschnitt 3.5). Dadurch stehen sowohl zu Beginn als auch zum Ende des Forschungsprojekts jeweils neun Monate für die Vor- und Nachbereitung zur Verfügung (s. Abb. 1).

Für ein dreijähriges Forschungsprojekt mit zweistufigem Antragsverfahren sind vier Veranstaltungen zu empfehlen. Je nachdem kann zu den bereits genannten Veranstaltungen optional Veranstaltung 2: Weg-Bereiterin (s. Abschnitt 3.2) oder Veranstaltung 4 Ziel-Erspäherin (s. Abschnitt 3.4) eingesetzt werden (s. Abb. 2). Die Weg-Bereiterin ermöglicht eine Rückkopplung über die Ausrichtung des Projekts mit externen Akteuren und die Integration von deren Wissen noch während der Orientierungsphase. Die Ziel-Erspäherin dagegen unterstützt die gemeinsame Wissensproduktion und betont somit die Arbeitsphase. Aufgrund des zweistufigen Antragsverfahrens muss die Einstiegsphase kürzer ausfallen, so dass sie bereits nach vier Monaten durch die erste Veranstaltung abzuschließen ist. Entsprechend verbleiben nach der letzten Veranstaltung noch fünf Monate für die Finalisierung des Forschungsprojekts.

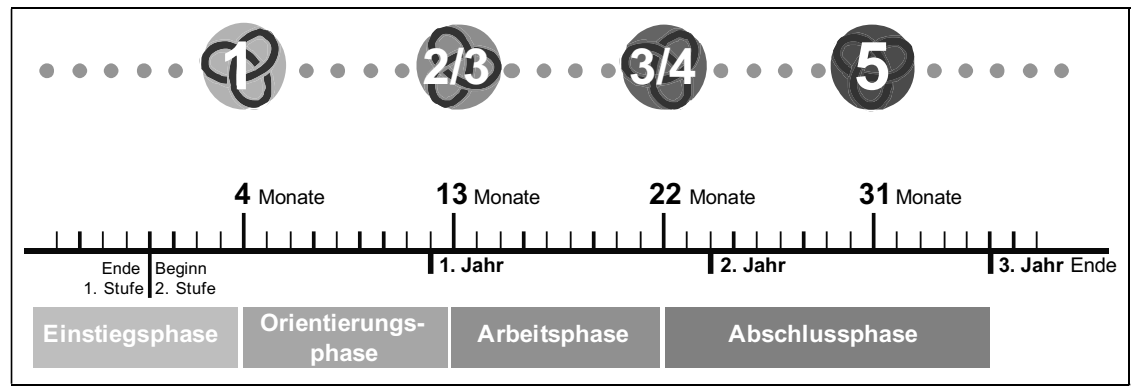

Abbildung 2: Zeitplan eines dreijährigen Forschungsprojekts mit vier Veranstaltungen. (C) Reallabor SRB. 


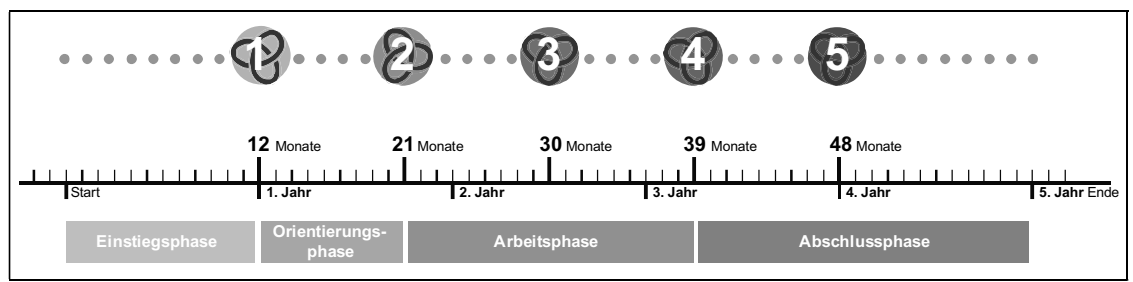

Abbildung 3: Zeitplan eines fünfjährigen Forschungsprojekts mit fünf Veranstaltung. (C) Reallabor SRB.

Für ein fünfjähriges Forschungsprojekt sind fünf bis sechs Veranstaltungen möglich (s. Abb. 3 und 4). Somit wird jede Projektphase durch mindestens eine Veranstaltung definiert, wobei die Arbeitsphase durch zwei oder drei Veranstaltungen gegliedert werden kann, das heißt, Veranstaltung 4: Ziel-Erspäherin (s. Abschnitt 3.4) wird bei insgesamt sechs Veranstaltungen doppelt eingesetzt (s. Abb. 4). Durch die Dopplung ist es möglich, jeweils verschiedene Themenschwerpunkte für die gemeinsame Wissensproduktion mit den externen Akteuren zu setzen.

Eine zusätzliche, sechste Veranstaltung wird insbesondere für Forschungsprojekte mit einem zweistufigen Antragsverfahren empfohlen. Bei fünf Veranstaltungen ergibt sich eine Einstiegsphase (und damit Vorbereitungszeit) von einem Jahr bis zur ersten Veranstaltung. Gleiches gilt für die Abschlussphase am Ende. Bei sechs Veranstaltungen verkürzt sich die Zeit bis zur ersten Veranstaltung auf sieben Monate, und am Ende verbleiben noch acht Monate für die Finalisierung des Forschungsprojekts.

Durch den bewussten Einsatz der Veranstaltungen als Meilensteine zur Definition der Projektphasen sowie durch die entsprechende Kommunikation nach innen und außen wird die Strukturierung des Forschungsprozesses sowohl für das interne Projektteam als auch für die externen Akteure transparent und nachvollziehbar.

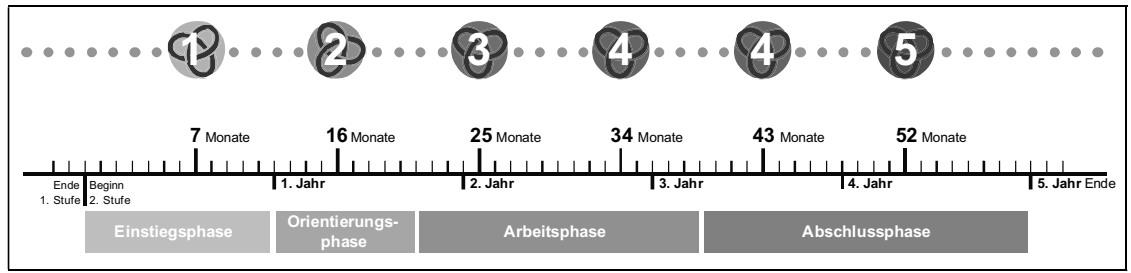

Abbildung 4: Zeitplan eines fünfjährigen Forschungsprojekts mit sechs Veranstaltungen. (C) Reallabor SRB. 


\section{Schritt 2: Definition von Ort, Raum und Zeit der Veranstaltungen}

Jede Veranstaltung ist zweitägig, wobei das Symposium am ersten Tag und der Reflexionsworkshop am zweiten Tag stattfinden. Es empfiehlt sich, bereits zu Beginn des Projekts festzulegen, wo und wann genau die Symposien und internen Reflexionsworkshops stattfinden sollen. Neben der frühzeitigen Suche und Reservierung geeigneter Räumlichkeiten ist dabei vor allem relevant, dass die Veranstaltungsorte für alle Beteiligten (interne wie externe) gut erreichbar sein müssen. Bei der Festlegung des Zeitpunkts sind Wochentag und Uhrzeiten von Beginn und Ende mit besonderer Sorgfalt unter Berücksichtigung der relevanten internen und externen Akteure aus Wissenschaft und Praxis zu wählen. Die Parameter bezüglich Ort, Raum und Zeit sollten möglichst zu Beginn des Forschungsprojekts oder bereits während der Antragstellung definiert werden, weil sie ein wesentlicher Bestandteil der Forschungsplanung und der Budgetierung sind.

\section{Schritt 3: Inhaltliche Vorbereitung jeder Veranstaltung als Meilenstein}

Die Veranstaltungen sind Meilensteine und dienen der regelmäßigen Standortbestimmung innerhalb des Forschungsverlaufs. Das heißt, die Forscher(innen) müssen für jeden Meilenstein ihren aktuellen Stand des jeweiligen Teilprojekts und des Gesamtprojekts dokumentieren und präsentieren können. Dieser Stand findet Eingang in die Vorträge der Symposien. Dafür müssen die Vorträge in einer Sprache formuliert werden, die für alle externen Akteure verständlich ist. Zudem werden der Stand der Arbeit, aktuelle Schwierigkeiten sowie die geplanten weiteren Schritte im internen Reflexionsworkshop dargelegt und gemeinsam besprochen. Die dadurch entstehende Pflicht einer regelmäßigen Reflexion, Aufbereitung und Berichterstattung unterstützt die Steuerung des Forschungsprozesses und die inhaltliche Vernetzung der Teilprojekte. Damit die Veranstaltungsreihe diese Funktion erfüllen kann, gilt es, die Erwartungen an die Vorbereitung der einzelnen Veranstaltungen im Antrag oder spätestens zu Beginn der Einstiegsphase auszuformulieren.

\section{Schritt 4: Dokumentation der Veranstaltungen}

Im Rahmen der einzelnen Veranstaltungen gilt es, ,Produkte ‘ und Diskussionen des Symposiums sowohl fotografisch als auch mittels Ton- und Videoaufnahmen $\mathrm{zu}$ dokumentieren, um sie als (gemeinsam) generiertes Wissen in die Forschung einfließen zu lassen und um sie für Pressearbeit und Dokumentationszwecke zu nutzen, z. B. für Präsentationen des Projekts auf der Webseite. Im Zuge der Projektplanung sind ggf. entsprechende forschungsethische Genehmigungen einzuholen, und es ist festzulegen, wie das diesbezügliche Einverständnis der Teilneh- 
mer(innen) eingeholt und dokumentiert wird. Die genaue Art der Dokumentation wiederum ist abhängig von den Methoden, die während der Veranstaltungen eingesetzt werden. Näher erläutert wird die Dokumentationsart im Folgenden jeweils in den Beschreibungen der einzelnen Veranstaltungen unter Aufgabenpaket 6 „Dokumentieren und Auswerten“ in den Abschnitten 3.1-3.5. Die Dokumentation der internen Reflexionsworkshops wiederum erfolgt nur zur internen Verwendung. Sie erfolgt, indem protokolliert wird, was im Team erarbeitet und besprochen wird. Auch darauf wird, soweit Bedarf besteht, jeweils unter Aufgabenpaket 6 ,Dokumentieren und Auswerten“ näher eingegangen.

\section{Schritt 5: Erstellung des Projektplans (Zusammenfassung)}

Aus den zuvor erläuterten Meilenstein- und Verknüpfungsfunktionen der nun ins Auge gefassten Exchange-Veranstaltungsreihe ergibt sich der Projektplan zur Durchführung der transdisziplinären Arbeit und der Synthesebildung im Forschungsprojekt. Abb. 5 zeigt einen idealisierten Projektplan für ein Forschungsprojekt, das aus zwei Teilprojekten besteht. Senkrecht in der Mitte ist die Veranstaltungsreihe, die dem Ganzen Halt und Struktur gibt. Die fünf Veranstaltungen bilden die Verknüpfungspunkte der Wissensintegration. Die Pfeile stellen die Wissensbestände und Impulse dar, die aus den Teilprojekten in die Veranstaltungen einfließen. Zusätzlich zu den Inhalten der Teilprojekte wirken auch die Erfahrungen der externen Akteure aus Wissenschaft und Praxis sowie die Dynamik im Praxisfeld auf die Veranstaltungen ein (gepunktete Pfeile). Die Erkenntnisse aus den Veranstaltungen wiederum fließen zurück in die Teilprojekte. Die Grafik veranschaulicht, wie das Design eines transdisziplinären Forschungsprojekts zu denken ist, wenn es mit einer Exchange-Veranstaltungsreihe strukturiert wird. Bei der Erstellung des Projektplans für ein konkretes Projekt sind die Wissensbestände und Impulse, die in der Grafik durch die Pfeile nur symbolisiert werden, inhaltlich zu beschreiben. Diese Inhalte sind im Projektverlauf regelmäßig fortzuschreiben. 


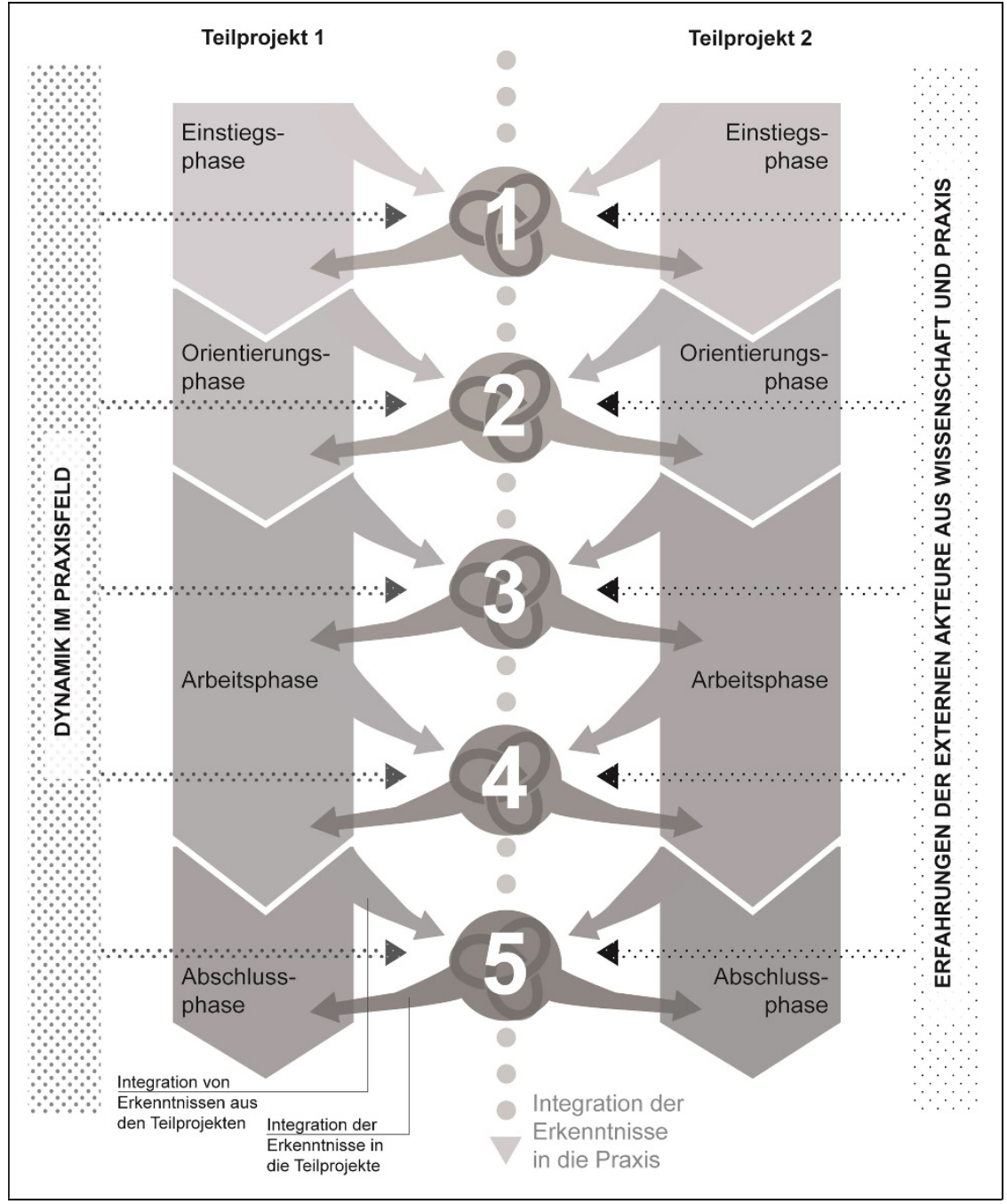

Abbildung 5: Idealisierter Projektplan, bei dem eine Exchange-Veranstaltungsreihe das ,Gerüst‘ für die Forschungsplanung bildet. (C) Reallabor SRB. 


\section{Die einzelnen Veranstaltungen der Exchange-Veranstaltungsreihe}

In diesem Kapitel wird die Planung der einzelnen Veranstaltungen der ExchangeVeranstaltungsreihe dargestellt. Jede Veranstaltung setzt einen eigenen Schwerpunkt und dient spezifischen Zielen (s. Abschnitte 3.1-3.5). Sie besteht immer aus zwei Teilen: einem öffentlichen Symposium am ersten Tag und einem internen Reflexionsworkshop am folgenden Tag (zu letzterem s. Abschnitt 3.6). In der Exchange-Veranstaltungsreihe werden fünf Veranstaltungen unterschieden, deren Kombination abhängig von der Dauer und den Schwerpunkten eines Projekts festgelegt wird (s. Abschnitt 2.2), wobei Veranstaltung 2 und Veranstaltung 4 bei einem dreijährigen Forschungsprojekt optional sind:

- Veranstaltung 1: Tür-Öffnerin (Abschnitt 3.1)

- Veranstaltung 2: Weg-Bereiterin (optional) (Abschnitt 3.2)

- Veranstaltung 3: Weg-Begleiterin (Abschnitt 3.3)

- Veranstaltung 4: Ziel-Erspäherin (optional) (Abschnitt 3.4)

- Veranstaltung 5: Ziel-Beschreiterin (Abschnitt 3.5)

Gemeinsam sind allen Veranstaltungen der grundlegende zeitliche Aufbau, die Intention des Reflexionsworkshops sowie, dass die Symposien und die Reflexionsworkshops dokumentiert werden (s. Kapitel 2).

Während alle Symposien einen ähnlichen Start haben, unterscheiden sie sich nicht nur hinsichtlich ihrer Ziele und Themen, sondern auch hinsichtlich der eingesetzten Beteiligungsmethoden (s. zu den Unterschieden auch Tab. 2 in Abschnitt 3.7). Aus diesen Unterschieden ergeben sich innerhalb von sechs immer gleichen Aufgabenpaketen spezifische Planungsaufgaben für jede Veranstaltung. Diese sechs Aufgabenpakete (AP) sind:

- AP 1: Identifizieren und Erreichen der Akteure

- AP 2: Inhaltliche Impulse aus dem Projekt bestimmen

- AP 3: Vorbereitungen speziell für externe Akteure treffen

- AP 4: Beteiligungsmethodik definieren

- AP 5: Wertschätzung durch Atmosphäre und Stimmung vermitteln

- AP 6: Dokumentieren und Auswerten 
Im Folgenden werden Ausrichtung und Planung der fünf Veranstaltungen beschrieben. Wobei letzteres anhand dieser sechs Aufgabenpakete erfolgt (bei AP 4 jeweils ergänzt um ein Beispiel aus dem Reallabor SRB, s. SRB-Boxen).

\subsection{Veranstaltung 1: Tür-Öffnerin}

Zur Gewinnung von Praxispartnern bedarf es einer Vorstellung der Inhalte und Ziele des Forschungsprojekts, um dieses zu bewerben und Praxisakteure über die aktuellen wissenschaftlichen Fragestellungen rund um das Projektthema zu informieren. Hier setzt die Veranstaltung $1 \mathrm{zu}$ Beginn des Projektverlaufs (Einstiegsphase) an. Ziel dieser Veranstaltung ist zum einen die Gewinnung und Einbindung von (weiteren) Praxisakteuren für die und in die transdisziplinäre Zusammenarbeit und Generierung von Wissen (s. zur Generierung von Wissen Eckart et al. 2018, S. 116). Zum anderen ist es die Vorstellung des Projektteams und dessen Expertise. Aus der Projektperspektive besteht der Hauptnutzen dieser Veranstaltung in der Legitimierung des Projekts als Akteur in der Forschungs- und Praxislandschaft sowie im Aufbau eines Netzwerks für einen späteren Austausch. Zudem wird der Grundstein für eine vertrauensvolle und kooperative Zusammenarbeit im Projekt gelegt.

\subsubsection{Steckbrief Tür-Öffnerin}

Wissensintegration und Bildungsziele: Durch den Einsatz dieser Veranstaltung wird insbesondere das Zielwissen externer Akteure in das Projekt und dessen Zielstellungen integriert. Externe Akteure (Praxisakteure, externe Forscher(innen), Student(inn)en, ggf. weitere Personen) werden sowohl informiert als auch konsultiert. Gleichzeitig profitieren diese von einem persönlichen Wissenserwerb sowie vom Aufbau neuer Kontakte und vom Austausch nicht nur mit dem Projektteam, sondern auch untereinander.

Ablauf und Rollen: Zu Beginn geben Kurzvorträge aus dem Projektteam Einblicke in das Forschungsprojekt. Anschließend erhalten die Teilnehmer(innen) die Möglichkeit, sich selbst ein Bild des Projekts zu machen und mit dem Projektteam zu diskutieren. Rolle der verschiedenen Akteure:

- Moderation: Projektteam

- Impulsreferate: Forscher(innen) aus dem Projektteam

- Teilnehmer(innen): Nehmen als interessiertes Publikum teil, haben darüber hinaus keine spezifische Rolle

Nutzen für externe Praxisakteure:

- Erweiterung und Pflege ihres Netzwerks, Austausch mit Peers

- Horizonterweiterung, Erwerb neuen Wissens 
Fragen, die die Inhalte der Tür-Öffnerin definieren: Was ist das Forschungsthema? Was ist das Forschungsvorhaben? Wer ist das Projektteam? Welche Expertise bringt das Projektteam mit? Welche Rolle wird das Projektteam im Feld einnehmen? Wie reagieren die externen Akteure auf das Projekt?

Voraussetzungen, die für den Einsatz der Tür-Öffnerin erfüllt sein müssen:

- Projektteam steht fest.

- Forschungsthemen und Fragestellungen sind definiert.

- Relevante Akteure im Feld (Praxis und Forschung) sind identifiziert.

\subsubsection{Planungsaufgaben Tür-Öffnerin}

AP 1 (Identifizieren und Erreichen der Akteure): Das Projektteam führt eine Akteursanalyse durch (s. Eckart et al. 2018, S. 120ff.; von Interesse sind sowohl Praxisakteure als auch Forscher(innen), die nicht im Projektteam involviert sind). Um die Akteure bestmöglich zu erreichen, werden verschiedene Formen der breiten Bewerbung des Symposiums eingesetzt: persönliche Einladungen per Post; Einladungen per E-Mail; Ankündigen über Online-Portale sowie auf den Webseiten der beteiligten Institutionen; Aushänge und Flyer in Hochschulen, Schulen, Läden, Gemeindeverwaltungen etc.

AP 2 (Inhaltliche Impulse aus dem Projekt bestimmen): Vertreter(innen) des Projektteams geben Einblicke in die Forschung zum Thema und stellen das Projekt, die Relevanz der Forschung und die Forschungsfragen dar. Sie erläutern die Projektstruktur und die Arbeitsweise im Projektteam. Die Nutzung von Zeitungsartikeln und prägnanten Zitaten aus der Presse dienen dazu, die Relevanz und die Aktualität der Thematik herauszustellen. In der Planung gilt es, die Elemente und Botschaften zu identifizieren, die präsentiert werden sollen, und dazu Konsens im Projektteam herzustellen.

AP 3 (Vorbereitungen speziell für externe Akteure treffen): Vorträge von Expert(inn)en aus dem Projektteam können als Publikumsmagnet fungieren, sofern bekannte Forscher(innen) oder Praxispartner zum Team gehören. Dies dient sowohl dazu, die Attraktivität der Veranstaltung zu steigern, als auch dazu, die Legitimierung und Anerkennung des Projekts als Ganzes beziehungsweise der Beteiligten als Expert(inn)en im Themenbereich zu erhöhen. Fehlt im Team ein solcher ,Magnet', gilt es zu überlegen, mit welchen anderen Mitteln diese erste Veranstaltung für externe Akteure besonders attraktiv gemacht werden könnte.

AP 4 (Beteiligungsmethodik definieren): Wichtig ist es bei dieser Veranstaltung, einen Ausgleich zu schaffen zu den passiv-aufnehmenden Formen der Kommunikation, d. h., Raum zu schaffen und Zeit zu bieten für informelle Gespräche und spontane Begegnungen. Eine gute Möglichkeit, sich selbst ein Bild von dem Pro- 
jekt zu verschaffen, bietet eine Ausstellung (siehe SRB-Box 1). Während Vorträge und Diskussionsrunden Inhalte und den Rhythmus vorgeben, eignet sich zur ,Aktivierung' der Teilnehmer(innen) eine Beteiligungsmethode, die speziell den Austausch der Teilnehmer(innen) untereinander und mit dem Projektteam anregt sowie den Rhythmus des Geschehens den Teilnehmer(inne)n anpasst. Für die Tür-Öffnerin eignet sich im Besonderen ein Markt der Möglichkeiten. Dies ist ein offenes Format, das allen Beteiligten einen Raum für selbstgesteuerte Kommunikation bietet. Der informelle Charakter der ,Marktstände', die verschiedene Aspekte des Forschungsthemas und Projekts zeigen, befördert eine entspannte Atmosphäre, die zum Austausch zwischen allen Beteiligten einlädt. Auf diese Weise können zum einen Forschungsinhalte individuell kennengelernt werden, zum anderen erhalten die einzelnen Akteure die Möglichkeit, sich persönlich untereinander zu vernetzen.

SRB-Box 1: Sich gegenseitig kennen und schätzen lernen in entspannter Atmosphäre: Akteure unterhalten sich über denkbare Ansätze für die Weiterentwicklung von Schulen

Im Reallabor SRB wurde eine Ausstellung als Markt der Möglichkeiten organisiert, um das Projekt im Rahmen des Symposiums zu präsentieren (s. Abb. 6). Der Markt der Möglichkeiten konnte vor, während und nach dem Symposium von den teilnehmenden externen Akteuren besucht werden. An Posterwänden, die thematisch den einzelnen Elementen des Projekts zugeordnet waren, standen Mitglieder des Projektteams für Diskussionen bereit. Sie kamen mit den ,Marktbesucher(inne)n' ins Gespräch und konnten sich so mit diesen über das Forschungsvorhaben, die Relevanz der Fragestellungen in der Praxis sowie mögliche weitere relevante, aber im Projekt noch nicht enthaltene Aspekte austauschen. Es wurden Arbeiten eines studentischen Wettbewerbs ausgestellt, die mögliche Ansätze für ein Transformationsvorhaben einer Schule zeigten, in Form von Plänen und sinnlich erfahrbaren dreidimensionalen Modellen. Die Student(inn)en präsentierten dazu selbst an ihrem ,Marktstand'ihre Ideen und standen für Fragen Interessierter bereit. Die interessengesteuerten Diskussionen zwischen Teammitgliedern und ,Marktbesucher(inne)n' stellten einen niederschwelligen Zugang zu dem Thema dar und unterstützten eine lockere Atmosphäre. Der Markt der Möglichkeiten wurde im Programm mit einer längeren Pause verbunden. Durch die Nähe der ,Marktstände' zu Stehtischen, an denen gegessen werden konnte, entstand ein ,reges Treiben', und zusätzlich unterstützte die Verbindung von Verpflegung und Diskussion eine entspannte Atmosphäre. Um der Pause eine Struktur zu verleihen, wurde ein Gong (analog zur Schulglocke) eingesetzt. Dieser ertönte drei Mal, nach jeweils 15 Minuten. Wer wollte, konnte dann an einen anderen ,Marktstand' oder Stehtisch zum informellen Gespräch wechseln. Dieser Gong ermöglichte eine zwanglose Strukturierung der Pause, so dass sich die Teilnehmer(innen) zurückziehen und später wieder zum ,Marktgeschehen“ dazustoßen konnten. An einzelnen ,Marktständen' gab es auch kurze Vorträge, die jeweils kurz nach dem Gongschlag begannen. Die Veranstaltung stellte so einen gelungenen Einstieg als ,Tür-öffner' in das Forschungsthema und das Forschungsprojekt für die externen Akteure dar. 

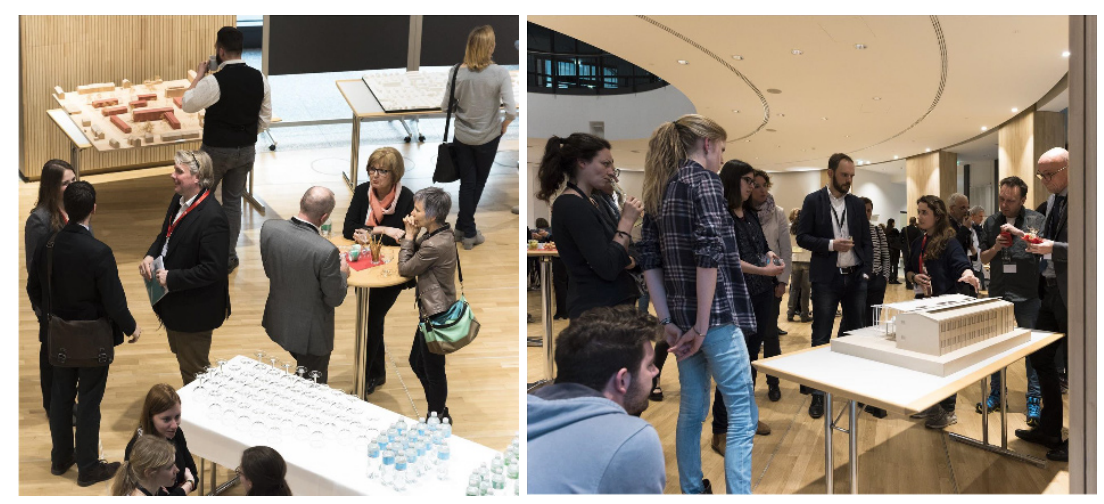

Abbildung 6: Austausch zwischen den Teilnehmer(inne)n und Teammitgliedern auf dem Markt der Möglichkeiten der Veranstaltung „Old School - Neues Lernen Vol. 2" des Reallabors SRB. Die auch räumliche Verbindung von Verpflegung und Diskussion erlaubte nicht nur eine Vielzahl von Gesprächen, sondern auch eine individuell gesteuerte Auseinandersetzung mit dem Thema. (C) Reallabor SRB 2017.

AP 5 (Wertschätzung durch Atmosphäre und Stimmung vermitteln): Die erste Veranstaltung dient dem Aufbau eines abgegrenzten Ortes, der Vertrauen und Offenheit unter allen Akteuren schafft. Da die Kommunikationsform den Charakter der Situation prägt, eignet sich ein Markt der Möglichkeiten in besonderer Weise, weil er sich dazu anbietet, eine offene Beteiligung sicherzustellen und soziale Ausschlussformen zu vermeiden (s. zur Wahl der Methoden Bergold und Thomas 2010, S. 338; zu offener Beteiligung/Transparenz Benighaus und Renn 2016, S. 80ff.). In der Durchführung ist ein besonderes Augenmerk auf Teilnehmer(innen) zu richten, die ,verloren' herumstehen. Für solche Fälle gilt es, bei der Planung eine Vorgehensweise und Zuständigkeiten zu vereinbaren.

AP 6 (Dokumentieren und Auswerten): Für die Ergebnisdokumentation des Markts der Möglichkeiten eignen sich Videoaufnahmen und Fotos (s. zum Einsatz solcher Dokumentationsmethoden Hofmann 2014, S. 45ff.). Fotos dienen im Falle der Tür-Öffnerin vorwiegend der Dokumentation der Veranstaltung mit Blick auf die Kommunikation nach außen. Um die inhaltlichen Ergebnisse zu erfassen, bieten sich vor allem Gedächtnisprotokolle an, die im Nachgang von jenen verfasst werden, die an den ,Marktständen“ als Ansprechpersonen bereitstanden. In der Planung gilt es, Kriterien und die inhaltliche Stoßrichtung dieser Protokolle festzulegen. 


\subsection{Veranstaltung 2: Weg-Bereiterin (optional)}

Gezielt die Erfahrungen, Erkenntnissen und Herausforderungen der externen Praxisakteure einzuholen und zu diskutieren, erlaubt es, die Praxisrelevanz der Forschungsthemen $\mathrm{zu}$ erhöhen, indem diese mit den Bedürfnissen der Praxis abgestimmt werden. Dem dient in der Orientierungsphase (zwischen Anfang und Mitte des Projektverlaufs) die Veranstaltung 2. Ziel dieser Veranstaltung ist es, Praxisakteure im Forschungsprozess mitzunehmen und die Forschungsfragen auf die aktuelle Praxissituation anzupassen. Der Nutzen dieser Veranstaltung für das Projekt liegt also vor allem darin, die Bedürfnisse der Praxis wahrzunehmen. Da$\mathrm{zu}$ ist eine transparente Darstellung des Forschungsstands notwendig, die ergänzt wird um Impulsvorträge von Praxispartnern, die die Teilnehmer(innen) aus ihrer Perspektive über die Auswahl und Begründung der einzelnen Schwerpunkte im Projekt informieren, über die Forschungsmethoden sowie ggf. die Auswahl und Bedeutung der Fallbeispiele.

\subsubsection{Steckbrief Weg-Bereiterin}

Wissensintegration und Bildungsziele: Durch den Einsatz dieser Veranstaltung werden Wissen und Erfahrungen der Praxispartner (z. B. aus den im Projekt behandelten Fallbeispielen), die im besten Fall durch eigene vorausgegangene Transformationsprozesse bereits Erfahrungen sammeln konnten, zur Verfügung gestellt. Dieses Wissen kann inner- wie außerhalb des Projekts sowohl in der Forschung als auch in der Praxis integriert werden. Die anwesenden Forscher(innen) können ihre Kompetenzen mithilfe der Lösungsansätze aus der Praxis entwickeln, und umgekehrt können die anwesenden Praxisakteure ihre Kompetenzen bezogen auf das Wissen der im Projekt vertretenen (inter-)disziplinären Perspektiven verfolgen.

Ablauf und Rollen: Im ersten Symposiumsteil geben Vorträge den Teilnehmer(inne)n Einblicke in Forschung und Praxis. Im zweiten Teil werden die Teilnehmer(innen) mit in die Diskussion einbezogen. Raum für Gespräche in kleineren Gruppen bietet diesmal ein anschließendes Catering. Rolle der verschiedenen Akteure:

- Moderation: Projektteam

- Impulsreferate: Praxispartner (wenn möglich solche, die Fallbeispiele vertreten)

- Teilnehmer(innen): Nehmen an einer gesteuerten Diskussionsrunde teil

Nutzen für externe Praxisakteure:

- Pflege ihres Netzwerks, Austausch mit Peers

- Einblicke in die Perspektive und Erfahrungen Dritter, daraus Impulse für die eigene Arbeit 
Fragen, die die Inhalte der Weg-Bereiterin definieren: Wie wird geforscht? Wie soll der Forschungsprozess aussehen? Welche Methoden sollen eingesetzt werden (ggf.: Welche Fallbeispiele bilden den Untersuchungsgegenstand?) und sind diese geeignet?

Voraussetzungen, die für den Einsatz der Weg-Bereiterin erfüllt sein müssen:

- Falls mit Fallbeispielen gearbeitet wird: Fallbeispiele für die Forschung sind ausgewählt.

- Erste Forschungserfahrungen, erste Transformationserfahrungen liegen vor.

\subsubsection{Planungsaufgaben Weg-Bereiterin}

AP 1 (Identifizieren und Erreichen der Akteure): Der Kreis der Teilnehmer(innen) entspricht dem Kreis, der für Veranstaltung 1 eingeladen wurde. Falls Veranstaltung 2 realisiert wird, kann der Kreis gezielt um Akteure erweitert werden, die in Veranstaltung 1 , gefehlt' haben.

AP 2 (Inhaltliche Impulse aus dem Projekt bestimmen): In der Veranstaltung stellen Praxispartner ihre für das Projekt relevanten Fallbeispiele/Themen/Erfahrungen vor. Diese sollten so aufbereitet und vorgetragen werden, dass sie leicht verständlich und spannend erzählt werden und das deutlich wird, weshalb diese für das Projekt ausgewählt wurden. Letztendlich ist das Ziel der Vorstellung nicht nur, dass die Teilnehmer(innen) Einblicke in das Projekt gewinnen, sondern auch, dass sie von Erfahrungen, die aus existierenden Beispielen stammen, lernen und sie in ihre Kontexte übertragen können. In der Vorbereitung muss geklärt werden, welcher Fokus sich eignet, um das Wissen und die Erfahrungen der Teilnehmer(innen) zu aktivieren, die eine Anpassung der Forschung an die Praxis erlauben. Ein wichtiger Schritt ist die Wahl der Themenschwerpunkte bzw. Fallbeispiele für die Veranstaltung. Welche Fallbeispiele oder Themen sind eine gute Grundlage für die Darstellung der Forschungsfragen? Es ist nützlich, dafür, wie bereits bei Veranstaltung 1, die Praxispartner einzubinden, die besonders bekannt oder im Feld etabliert sind. Einen wichtigen Stellenwert hat das Briefing, damit die Impulse in das Symposium den gewünschten Fokus haben.

AP 3 (Vorbereitungen speziell für externe Akteure treffen): Fallbeispiele von Praxispartnern oder aus dem Projektteam können als Publikumsmagnet fungieren, sofern sie spannend aufbereitet und vorgetragen werden. Sie dienen sowohl dazu, die externen Praxispartner als Kooperationspartner stärker einzubinden, als auch, die Praxisnähe, Anwendbarkeit und Verständlichkeit des Projekts zu erhöhen. Gerade zu Projektbeginn, wenn erst wenige oder keine Ergebnisse vorliegen, steigern Fallbeispiele aus existierenden Projekten die Veranstaltungsattraktivität für externe Akteure und zeigen die Vernetzung zu unterschiedlichen Kontexten auf. 
AP 4 (Beteiligungsmethodik definieren): Die Praxispartner können ihre Fallbeispiele in Vorträgen präsentieren. Aber auch eine Ausstellung ist eine ansprechende Methode, um die Fallbeispiele/Themen/Erfahrungen den Teilnehmer(inne)n in visueller Form näherzubringen. Als Methode ebenfalls geeignet ist das sogenannte "Storytelling“, in dem das Fallbeispiel/Thema in eine ,Geschichte“ verpackt wird. Da die Praxisakteure auch in einen strukturierten Austausch treten sollen, werden sie nach den Präsentationen in die Diskussion mit einbezogen. Hierzu sind Beteiligungsformate sinnvoll, die es ermöglichen, Plenumspräsentationen damit zu verbinden, dass Teilnehmer(innen) ihre Erfahrungen einbringen und Rückmeldungen zu den Präsentationen geben (s. zum Diskurs sowie zum Vorgehen bei Beteiligungsformaten Renn und Benighaus 2003, S. 108ff.). Für die Weg-Bereiterin bietet sich daher zum Beispiel das Fishbowl-Format an (auch Aquarium- oder Innen-/Außenkreis-Methode genannt, für eine Beschreibung der Methode s. z. B. United Nations Human Rights 2012; s. SRB-Box 2). Dies ist ein offenes Format, das eine Diskussion vor und mit einem Plenum ermöglicht, dabei aber im Vergleich z. B. zur Podiumsdiskussion die Hierarchie zwischen Podiumsgästen und Publikum aufhebt, indem alle Anwesenden Teil eines Sitzkreises werden. Diese Methode kann eine unbefangene Kommunikation im ,Aquarium“ unterstützen. In der Diskussion können nicht nur die Beispiele der Referent(inn)en diskutiert werden, es ist auch möglich, den Fokus der Diskussion auf Fragen zu richten, wie die Erfahrungen aus der Praxis in der Forschung angewendet werden könnten, so dass die Diskussionsergebnisse in den laufenden Forschungsprozess integriert werden können. Für eine noch stärker auf Wissensintegration zielende Methode ist zum Beispiel das Expertenpuzzle sinnvoll (auch Gruppenpuzzle oder Jigsaw-Methode genannt, für eine Beschreibung der Methode s. z. B. https://www.jigsaw.org, zugegriffen am 30.04.2019), das sich für die zunächst getrennte und dann integrierende Erarbeitung von Wissen zu einem Thema eignet. In einem ersten Schritt arbeiten dabei Praxisakteure in Gruppen zu jeweils einem Teilthema zusammen. Danach werden gemischte Gruppen gebildet, in die jeweils mindestens ein Praxisakteur eines jeden Teilthemas eingebunden ist, und diese Gruppen fügen die Erkenntnisse zu allen Teilthemen zusammen.

\section{SRB-Box 2: Auseinandersetzung auf gleicher Augenhöhe in großer Runde: Durch den Einbezug verschiedener Expertisen entstehen kontroverse Diskussionen zu aktuellen Schulentwicklungen}

In einem der Symposien des Reallabors SRB wurde für die Diskussionsrunde mit Praxisakteuren die Fishbowl-Methode angewandt (s. Abb. 7). Übergreifendes Thema der Diskussion war die Frage „Architektur und Schulkonzepte - eine Symbiose?“ Für den Einstieg in das Thema hielten eine Schulleiterin, ein Schulleiter und ein Architekt Impulsvorträge. Es handelte sich hierbei um Praxispartner aus dem Projekt, die gleichzeitig Vertreter(innen) der im Projekt bearbeiteten Fallbeispiele waren. Sie präsentierten ihre Erfahrungen in Bezug auf die von ihnen angewandten innovativen pädagogischen Kon- 
zepte bzw. auf die Tätigkeit als Schulbauberater. Diese praxisnahen Vorträge boten für die Teilnehmer(innen) einen leichten Einstieg, um die Themen schnell zu erfassen. Nach den Impulsvorträgen ging die Diskussion in großer Runde unter Einbezug des Plenums weiter. Das Format Fishbowl, das als innerer Sitzkreis von Hockern und als mehrere äußere Sitzkreise mit Stühlen eingerichtet war, bot für alle Beteiligten die Möglichkeit, sich unkompliziert einzubringen, Nachfragen zu stellen oder Ergebnisse zu kommentieren und auf die aktuelle Situation im Schulbau zu übertragen.

Zu Beginn der Diskussionsrunde nahmen die drei Referent(inn)en im inneren Kreis Platz und zwei Personen aus dem Projektteam setzten sich zusätzlich dazu. Sie erläuterten zuerst die ,Spielregeln' der Fishbowl und moderierten diese im weiteren Verlauf. Drei Hocker im inneren Kreis blieben für Praxisakteure aus dem Plenum frei. Diese freien Plätze füllten sich schnell, und viele der Teilnehmer(innen) wechselten während des Verlaufs zeitweise in den inneren Kreis, um sich an der Diskussion zu beteiligen. Sie stellten den Referent(inn)en und/oder dem Projektteam Fragen. Die Dynamik der Diskussion veränderte sich positiv, d. h. die Anzahl Fragen nahm im Verlauf der Diskussion deutlich zu, und die Zahl der Fragenden erweiterte sich kontinuierlich. Wertvoll an dieser Vorgehensweise war, dass die verschiedenen Perspektiven und Meinungsbilder der Akteure deutlich wurden und die unterschiedlichen Disziplinen sich untereinander ,erkannten', schätzen lernten und die Meinung der anderen antizipiert wurde. Durch die Methode wurde zudem deutlich, welche Impulse im Anschluss fokussiert und verdichtet werden sollten.

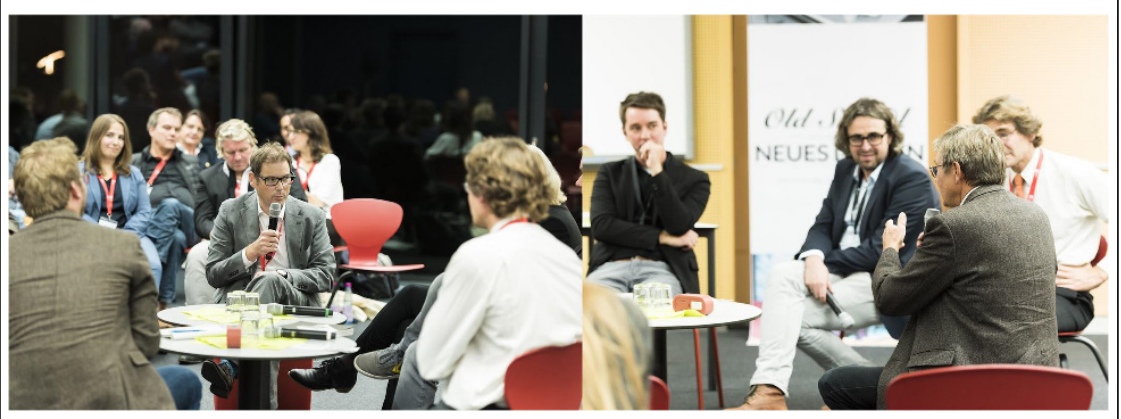

Abbildung 7: Sitzkreise mit Stühlen und Hockern bei der Fishbowl der Veranstaltung „Old School - Neues Lernen Vol. 3“. (C) Reallabor SRB 2017.

AP 5 (Wertschätzung durch Atmosphäre und Stimmung vermitteln): Durch die Impulse von Praxispartnern, die in einer alltagsnahen Sprache vorgetragen werden, kann die Brücke zu den Erfahrungen der Praxisakteure geschlagen werden. Kombiniert mit offenen, hierarchiefernen Diskussionsformaten (s. SRB-Box 2) erhöht dies die aktive Beteiligung der Teilnehmer(innen). Dies wiederum verstärkt die Wahrnehmung der Veranstaltung als Netzwerk- und Bildungsplattform. 
AP 6 (Dokumentieren und Auswerten): Um die Kommentare und Geschichten der Teilnehmer(innen) zu dokumentieren, eignen sich Ton- und Videoaufnahmen. Die Tonaufnahmen bzw. die Tonspur der Videoaufzeichnung sollten transkribiert und im Nachgang ausgewertet werden. Zusätzlich hilfreich sind Notizen zur Diskussion, die am nächsten Tag im Reflexionsworkshop direkt einfließen können. Fotos dienen im Fall der Weg-Bereiterin vorwiegend der Dokumentation der Veranstaltung für die Kommunikation nach außen. Im Reflexionsworkshop werden vor allem die Beiträge aus der Diskussion im Plenum ausgewertet. Die am Projekt Beteiligten nutzen die Feedbackschleife, um in dieser Projektphase nochmals die Themen zu priorisieren und den Projektfokus einzuengen. Damit dies gut gelingt, gilt es, sich im Vorfeld über die Art und Weise sowie den Fokus der Notizen während des Symposiums zu verständigen.

\subsection{Veranstaltung 3: Weg-Begleiterin}

Die Veranstaltung 3 liegt in der Mitte des Projektverlaufs, also in der Arbeitsphase. Ziel dieser Veranstaltung ist es, anhand der Darstellung des Forschungsstandes, d. h. der Erläuterung erster Forschungseindrücke und Erfahrungsberichte aus dem bisherigen Projektverlauf, die externen Akteure zum Nachdenken über die Projektergebnisse anzuregen, diese auf die eigene Arbeit zu übertragen und damit deren Anwendbarkeit zu bewerten. Ein besonderer Fokus liegt hier auf der Rückkoppelung mit dem wissenschaftlichen Diskurs. Diese Rückkopplung der Ergebnisse soll einen Beitrag leisten für eine innovative und dynamische Forschung, die auf die aktuelle Situation der Praxisakteure eingeht. Ziel und Nutzen ist insbesondere die Legitimierung des Projekts als Akteur in der Wissenschaft und ein Testen der Anwendungsorientierung der ersten Projektergebnisse.

\subsubsection{Steckbrief Weg-Begleiterin}

Wissensintegration und Bildungsziele: Veranstaltung 3 ,aktiviert ${ }^{\star}$ vor allem das Wissen der externen Forscher(innen), die durch ihre eigene Forschung bereits über Erfahrungen verfügen, die für das Projekt relevant sind. Dieses Wissen kann sowohl im Projekt als auch in der Praxis integriert werden. Gleichzeitig können damit alle Beteiligten ihr Wissen erweitern.

Ablauf und Rollen: In Inputs geben externe Forscher(innen) einen Überblick über das Forschungsfeld, während die internen Forscher(innen) anhand von Praxisbeispielen die Anwendung und Übertragbarkeit der ersten Forschungsergebnisse aus dem Projekt aufzeigen. Nach der, Vortragsrunde' bietet sich ein Beteiligungsformat an, das eine Übertragung der präsentierten Inhalte auf die Arbeit der Praxisakteure in gemischten Kleingruppen erlaubt. Rolle der verschiedenen Akteure: 
- Moderation: Projektteam, externe Forscher(innen)

- Impulsreferate: Vertreter(innen) des Projektteams, externe Forscher(innen)

- Teilnehmer(innen): Übertragung der Impulse aus der Forschung auf die eigene Arbeit im Praxisfeld

Nutzen für externe Praxisakteure:

- Pflege ihres Netzwerks, Austausch mit Peers

- Impulse aus der Forschung und Einblicke in die Forschung inner- und außerhalb des Projekts

- Impulse für das praktische Handeln in den eigenen Problemstellungen

Fragen, die die Inhalte der Weg-Begleiterin definieren: Wie ist der aktuelle Forschungsstand? Was sind die ersten Erkenntnisse und Erfahrungen, die das Projektteam gesammelt hat? Gibt es weitere Forschungsprojekte oder Initiativen im selben Themenbereich?

Voraussetzungen, die für den Einsatz der Weg-Begleiterin erfüllt sein müssen:

- Erste Erkenntnisse und Erfahrungen aus dem Projekt liegen vor.

- Es besteht Klarheit darüber, welches die für das Projekt besonders spannenden Forschungsfelder und -projekte sind, von denen gelernt werden kann.

\subsubsection{Planungsaufgaben Weg-Begleiterin}

AP 1 (Identifizieren und Erreichen der Akteure): Grundsätzlich wird derselbe Kreis an Akteuren eingeladen wie bei der/den vorangegangenen Veranstaltung(en). Zusätzlich gilt es, gezielt externe Forscher(innen) einzuladen, die dem Projekt spannende neue Impulse geben können. Im Hinblick darauf ist es von Nutzen, kontinuierlich und mit genügend Vorlauf eine Datenbank möglicher Referent(inn)en aufzubauen. Dafür bietet sich eine Literaturrecherche an, die einen Überblick über den aktuellen Stand der Forschung, aber vor allem auch über die aktiven und relevanten Akteure in der Forschungslandschaft gibt. Kriterien zur Auswahl von externen Forscher(inne)n müssen äußerst früh im Projektverlauf gemeinsam festgelegt werden.

AP 2 (Inhaltliche Impulse aus dem Projekt bestimmen): Vertreter(innen) aus dem Projektteam präsentieren den Zwischenstand und erste praxisrelevante Forschungsergebnisse des Projekts. Zur Vorbereitung gehört es, herauszuarbeiten, wie die mittlerweile vorliegenden Teilergebnisse aus dem Projekt so in Bezug zueinander gesetzt werden, dass den Teilnehmer(innen) ein Gesamtbild vermittelt werden kann. 
AP 3 (Vorbereitungen speziell für externe Akteure treffen): Um eine gute Mischung der Teilnehmer(innen) in den Kleingruppen zu unterstützen und ihre spezifische Expertise in Wert zu setzen, hilft es, die Teilnehmer(innen) nach Akteursgruppen ,aufzuteilen' sowie die Zuordnung der Individuen auf den Namensschildern durch kleine Aufkleber mit unterschiedlichen Farben auszuweisen. Die Aufteilung in unterschiedliche Akteursgruppen ist für das spätere Beteiligungsformat wichtig, um eine Mischung der Perspektiven, Erfahrungen und Expertisen zu gewährleisten. Die Zuordnung zu Akteursgruppen fällt leichter, wenn die Teilnehmer(innen) von Beginn an (also ab Veranstaltung 1) entsprechend kategorisiert erfasst werden, wenn also Daten zu den Teilnehmer(inne)n erhoben werden, die Auskunft über Parameter geben, die aus Projektsicht relevant sind.

AP 4 (Beteiligungsmethodik definieren): Um möglichst viele Praxisperspektiven fruchtbar zu machen, bietet sich an, in der Veranstaltung Weg-Begleiterin ein World Café umzusetzen (für eine Beschreibung der Methode s. Brown und Isaacs 2007; Benighaus und Benighaus 2016, S. $216 \mathrm{ff}$; http://www.theworldcafe.com, zugegriffen am 22.04.2019). Dieses Format nutzt die ungezwungene Kaffeehausatmosphäre, um die verschiedenen Teilnehmer(innen) einer Veranstaltung ins Gespräch zu bringen. Das Format kann gut eingesetzt werden, um die Anwendbarkeit der Forschungsergebnisse in der Praxis zu überprüfen. Tische mit jeweils unterschiedlichem Fokus geben die Themen, nach denen sich das Projekt inhaltlich strukturieren lässt, wieder. In der ersten Runde des World Cafés wird auf eine Mischung der Teilnehmer(innen) aus den unterschiedlichen Akteursgruppen geachtet (anhand der Farbcodierung der Namensschilder, s. dazu AP 3). In den folgenden Runden ist jedoch auch eine Zuordnung ausschließlich nach individuellen Neigungen möglich. Die Moderator(inn)en der Café-Tische (,Gastgeber(innen) ${ }^{6}$ ) übernehmen die Aufgabe, innerhalb der Tischgruppe die Diskussion in Fluss zu bringen und die Ergebnisse festzuhalten (s. SRB-Box 3). Mit dem Einsatz von Papiertischdecken, die als Schreibunterlage dienen, werden die Diskussionsbeiträge festgehalten. Die so dokumentierten Ergebnisse in Form von Skizzen, Zeichnungen und Mitschriften können in das Projekt einfließen, zitiert oder auch als solche übernommen werden.

\section{SRB-Box 3: Erste Projektergebnisse mit Praxisakteuren in Kleingruppen reflektieren und auf Anwendbarkeit überprüfen - Diskussion von Handlungsempfehlungen in der Schulbauplanung}

Für das Symposium zum Thema „Planungsprozesse von Schulen - neue Wege für neues Lernen" sollten Handlungsempfehlungen als erste praktische Ergebnisse diskutiert und kommentiert werden. Dazu wurde ein World Café eingesetzt (s. Abb. 8), mit dem eine Kaffeehausatmosphäre erzeugt wurde. Runde Tischinseln, die mit Papiertischdecken gedeckt wurden, dienten sowohl der Dekoration als auch als Schreibunterlage, um die wesentlichen Gedanken direkt mit Buntstift oder Marker festzuhalten. Blumenvasen auf 
den Tischen erzeugten einen informellen Charakter, der zum Verweilen einlud. Eine Café-Etikette, die die Kommunikationsregeln erläuterte, ermunterte die Teilnehmer(innen), ihre eigenen Ansichten und Ideen einzubringen und einander zuzuhören. Von den Teilnehmer(inne)n wurde diese entspannte Atmosphäre positiv wahrgenommen und der Raum zum offenen, ungezwungenen Gespräch sehr geschätzt.

Um die Tischgruppen möglichst heterogen zusammenzusetzen, wurden die Namensschilder anhand der Anmeldung kategorisiert. So erhielten beispielsweise alle Architekt(inn)en einen schwarzen Punkt auf dem Namensschild, alle Mitarbeiter(innen) kommunaler Verwaltungen einen grünen, alle Lehrer(innen) einen blauen Punkt und so weiter. Auf diese Weise konnten sich die Teilnehmer(innen) selbstständig in gemischte Gruppen zusammenfinden. Durch die Begrüßung der jeweiligen Tischgruppe durch die 'Gastgeber(innen)' und die kleine Gruppengröße von sechs bis acht Personen wurde ein intimer Raum geschaffen, um gemeinsam über das jeweilige Thema zu diskutieren. Es war zu beobachten, dass sich die Gruppen schnell in konstruktive Gespräche vertieften. Nach 30 Minuten gab es einen Wechsel der Gruppen. Die ,Gastgeber(innen)' fassten die Diskussion für die neue Gruppe, die sich am betreffenden Tisch einfand, zusammen. Auf diese Weise wurden die Themen und Fragen in den aufeinanderfolgenden Gesprächsrunden immer weiter vertieft. Aus Sicht der Diskussionsteilnehmer(innen) wichtige Überlegungen und Ideen wurden unverzüglich auf den Papiertischdecken festgehalten, um die Inhalte der Diskussion zu dokumentieren. Anschließend wurden im Plenum die Diskussionsergebnisse aller Thementische vorgestellt. Die beschriebenen Tischdecken wurden als Grundlage für den Reflexionsworkshop am folgenden Tag genutzt.

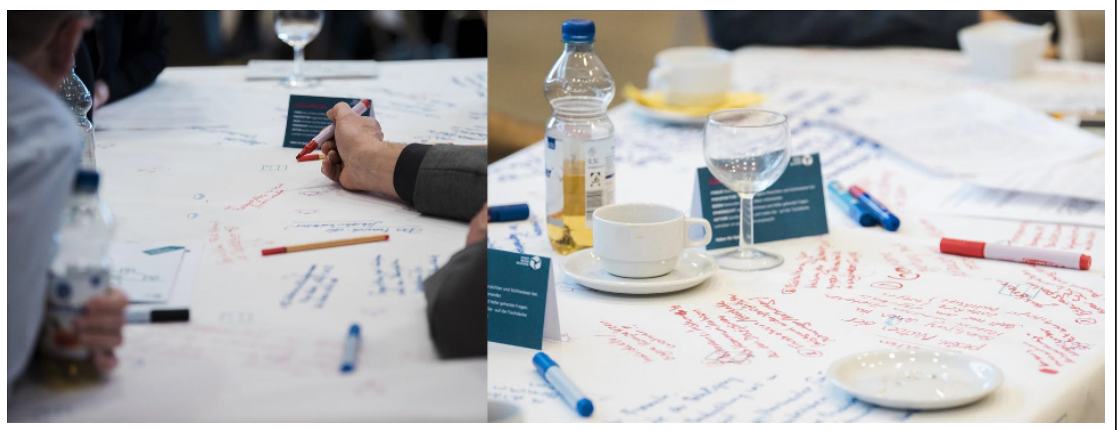

Abbildung 8: Papiertischdecken des World Cafés der Veranstaltung „Planungsprozesse von Schulen - neue Wege für neues Lernen“. Dadurch, dass die Ergebnisse spontan und von allen Diskussionsteilnehmer(inne)n aufgeschrieben werden, ist die Moderation besonders gefordert, weil sie den Gesprächsverlauf konzentriert verfolgen muss, um die Ergebnisse in nächsten World Café-Runden und dann im Plenum wiedergeben zu können.

(c) Reallabor SRB 2017. 
AP 5 (Wertschätzung durch Atmosphäre und Stimmung vermitteln): Ein World Café bietet einen guten Rahmen für einen offenen und ungezwungenen Austausch über die Projektergebnisse. Eine liebevolle Gestaltung des Cafés zeigt eine hohe Wertschätzung der Teilnehmer(innen) seitens der Verantwortlichen der Veranstaltung.

AP 6 (Dokumentieren und Auswerten): Im World Café dienen die Tischdecken der Dokumentation. Diese haben den Charakter von Protokoll-Notizen, die durch das Projektteam ausgewertet werden können. Im Reflexionsworkshop können die auf den Tischdecken festgehaltenen Ergebnisse direkt gesichtet und genutzt werden. Für eine spätere Auswertung hingegen ist es zwingend, ausführliche Protokolle zu erstellen, damit die Tischdecken-Notizen verständlich und die Ergebnisse der Diskussionen nutzbar werden. Dafür wiederum ist es sinnvoll, auch über Tonaufnahmen der an den Tischen geführten Diskussionen zu verfügen. Ebenfalls dienlich sind zeitnahe erstellte Zusammenfassungen des Diskussionsverlaufs an den Tischen durch die jeweiligen, Gastgeber(innen)' . Werden die Ergebnisse der Tische im Plenum vorgestellt und diskutiert, empfiehlt es sich zudem, dies durch Ton- und Videoaufnahmen zu dokumentieren.

\subsection{Veranstaltung 4: Ziel-Erspäherin (optional)}

Zwischen der Mitte und dem Ende des Projektverlaufs setzt Veranstaltung 4 während der Arbeitsphase ein. Diese Veranstaltung fördert vor allem die gemeinsame Wissensproduktion der beteiligten Akteure mit Blick auf die Nutzung der Ergebnisse in der Praxis. In der Weg-Begleiterin fand dieser Schritt schon im Ansatz statt, dieser wird aber in der optionalen Veranstaltung der Ziel-Erspäherin noch verstärkt, indem weitere Teilergebnisse aus dem Projekt vorgestellt und überprüft werden. Ziel der Veranstaltung ist eine systematische Rückkopplung der Erkenntnisse aus der Forschung an die Expertise der Praxisakteure. Zudem werden Praxisakteure, die bisher noch nicht im Netzwerk aufgetreten sind, gezielt eingeladen, um den Akteurskreis gegenüber den bisherigen Veranstaltungen zu erweitern.

\subsubsection{Steckbrief Ziel-Erspäherin}

Wissensintegration und Bildungsziele: In dieser Veranstaltung wird vor allem Transformationswissen der Praxisakteure für das Projekt fruchtbar gemacht, das in das Projekt integriert werden kann. Das Projektteam erwirbt so neues Wissen aus der Praxis. Der Wissensaustausch zwischen dem Projektteam und den externen Akteuren zu diesem Zeitpunkt, zu dem viele Ergebnisse vorliegen, stößt den gemeinsamen Lernprozess an. Diese Co-Produktion wiederum erleichtert es den 
Praxisakteuren, selbst einen persönlichen Wissenserwerb und eine Selbstentwicklung zu verfolgen, was auch ein Empowerment darstellt (s. dazu z. B. Eckart et al. 2018, S. 118).

Ablauf und Rollen: Der erste Teil der Ziel-Erspäherin wird durch Impulsvorträge zum Stand der Arbeit im Projekt eingeleitet. Im zweiten Teil der Veranstaltung werden in kleinen heterogenen Gruppen konkrete praxisbezogene Ansätze erarbeitet. Rolle der verschiedenen Akteure:

- Moderation: Projektteam oder extern beauftragte Moderation

- Impulsreferate: Vertreter(innen) des Projektteams

- Teilnehmer(innen): Entwickeln auf der Grundlage ihrer Expertise und für ihre Problemstellungen Ideen für mögliche Lösungen, indem sie Projektergebnisse auf ihre Situation anwenden

Nutzen für externe Praxisakteure:

- Pflege ihres Netzwerks, Austausch mit Peers

- Einblicke in und Impulse aus der Forschung

- Impulse für das praktische Handeln mit Bezug auf ihre Problemstellungen

- Empowerment und Hinweise, wie sie auch nach Ende des Projekts Transformationsprozesse gestalten können

Fragen, die die Inhalte der Ziel-Erspäherin definieren: Was sind die ersten Zwischenergebnisse der Forschung? Welche Erkenntnisse wurden bereits gewonnen? Wie werden bereits erhobene Daten bis zum Ende ausgewertet?

Voraussetzungen, die für den Einsatz der Ziel-Erspäherin erfüllt sein müssen:

- Wesentliche Teilergebnisse aus dem Projekt liegen vor

- Projektteam hat Überblick über Akteure im Feld, die bisher außen vor blieben, aber mit Blick auf die Praxisrelevanz und Legitimität der Ergebnisse beteiligt werden sollten.

\subsubsection{Planungsaufgaben Ziel-Erspäherin}

AP 1 (Identifizieren und Erreichen der Akteure): Grundsätzlich bleibt der Kreis der Teilnehmer(innen) derselbe wie bei den vorhergegangenen Veranstaltungen. In dieser Veranstaltung wird aber darüber hinaus ein Schwerpunkt in der Identifikation und ,Aktivierung' bisher zu wenig berücksichtigter Akteursgruppen gesetzt, die zusätzlich zum bisherigen Kreis gezielt angesprochen und eingeladen werden sollen. Besonderes Augenmerk liegt dabei auf marginalisierten Akteuren. 
Als ,marginalisierte Akteure" werden Akteure verstanden, die nicht über die Möglichkeiten oder Rahmenbedingungen verfügen, sich aktiv in das Forschungsprojekt einzubringen, beispielsweise Migrant(inn)en, Kinder, Senior(inn)en und andere. Die Frage in der Vorbereitung lautet also: Welche Akteursgruppen fehlen in der Betrachtung bis dato, und wie können diese erreicht werden? Durch die Auswertung der Teilnehmer(innen) der bisherigen Veranstaltungen entsteht ein Überblick über die schon beteiligten Akteure und das wiederum ist eine Grundlage zur Identifizierung der ,Lücken', die mit Blick auf eine umfassendere Beteiligung zu schließen sind.

AP 2 (Inhaltliche Impulse aus dem Projekt bestimmen): Vertreter(innen) aus dem Projektteam präsentieren Erkenntnisse aus dem Projekt, wobei vor allem solche ausgewählt werden, die aus Sicht des Teams ein besonderes Umsetzungspotenzial haben.

AP 3 (Vorbereitungen speziell für externe Akteure treffen): In dieser Veranstaltung gilt es, sorgfältig darauf zu achten, dass für marginalisierte Akteure die Materialien zielgruppenspezifisch vorbereitet werden.

AP 4 (Beteiligungsmethodik definieren): Um Kommunikationshindernisse zwischen dem Projektteam, bisherigen externen Akteuren und neu hinzukommenden marginalisierten Akteuren $\mathrm{zu}$ überwinden, werden niedrigschwellige Interaktionsebenen eingeführt und kreativ fördernde Methoden eingesetzt (s. zu solchen Methoden Hofmann 2014, S. 45ff.). Die gemeinsame Wissensproduktion kann beispielsweise durch die Anwendung der Methode Ideenwerkstatt angeregt werden (s. dazu Rogge 2000, S. 77ff.). Dieses Format motiviert die Teilnehmer(innen), nicht nur ins Gespräch zu kommen und intensiv zu diskutieren, sondern darüber hinaus gemeinsam Wissen und Ideen für die Praxis zu erzeugen (s. auch SRB-Box 4). Um kreativ arbeiten zu können, ist die Wahl des Raums für diese Veranstaltung besonders kritisch. Von Vorteil ist ein größerer Plenumssaal, in dem die Begrüßung, die Inputvorträge und die anschließende Präsentation der Gruppenergebnisse stattfinden kann. Zudem braucht es ausreichend Platz für die Gruppenarbeiten. Um zu verhindern, dass sich die Gruppen bei der Ideenwerkstatt gegenseitig stören, sollten die Werkstätten auf einer großen Fläche mit runden Stehtischen oder Sitzgruppen verteilt sein. Schließlich sollte der Raum ausreichend Platz für das Catering aufweisen. 


\section{SRB-Box 4: Ideenwerkstatt mit Kindern, Jugendlichen und Erwachsenen im Reallabor SRB: beliebte und unbeliebte Orte im Schulgebäude, auf dem Schulhof, auf dem Schulweg}

In einer Ideenwerkstatt mit Schüler(inne)n und Erwachsenen zum Thema „Beliebte und unbeliebte Orte in der Schule“ wurden im Rahmen des Symposiums „Lernen aus der Vergangenheit - Planen für die Zukunft" zwei getrennte Werkstätten durchgeführt (s. Abb. 9). Die Schüler(innen) arbeiteten in kleinen Sitzgruppen zusammen und konnten anhand von Fotografien ihre Lieblingsorte und ihre unbeliebten Orte im Schulgebäude, auf dem Schulhof und auf dem Schulweg beschreiben. Sie entwickelten anschließend Handlungsempfehlungen sowie mögliche Maßnahmen für den weiteren Umgang mit den unbeliebten Orten und zu deren Verbesserung. Sie schrieben die fünf wichtigsten Empfehlungen auf die fünf Seiten von Papphockern. Die Erwachsenen gingen ähnlich vor, hatten aber keine Fotos, die sie nutzen konnten. Sie beschrieben ebenfalls ihre beliebten und unbeliebten Orte im Schulgebäude, auf dem Schulhof und auf dem Schulweg, entwickelten Ideen zur Verbesserung und fassten ihre Ergebnisse in fünf Handlungsempfehlungen zusammen. Die Ergebnisse präsentierten die Schüler(innen) und Erwachsenen vor dem Plenum anhand der Papphocker, die sie in den Gruppen beschrieben hatten. Die entstandene Papphocker-Wand der Schüler(innen) und Erwachsenen zeigte zum Abschluss visuell die Ergebnisse und ließ die Vielfalt der Antworten deutlich werden.

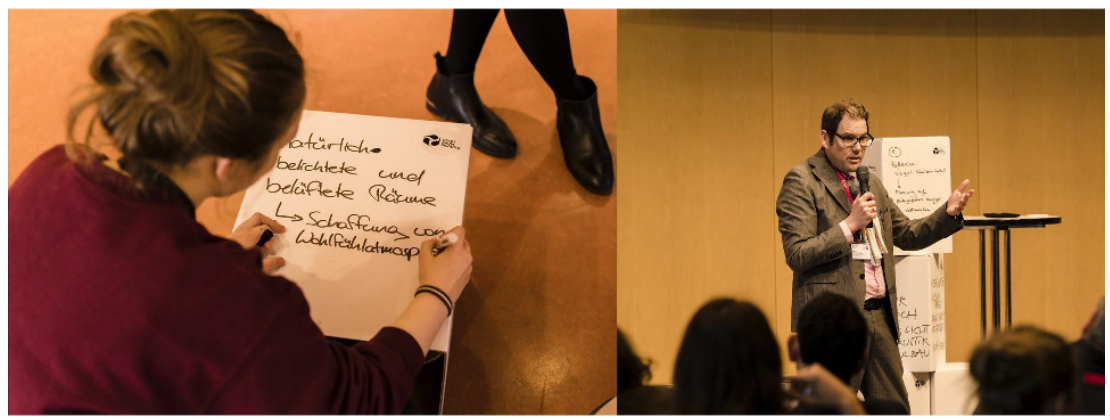

Abbildung 9: Gestaltung der Papphocker und Präsentation der Ergebnisse der Werkstätten auf der Veranstaltung „Old School - Neues Lernen Vol. 4“. Die ,dreidimensionale' Darstellung der Ergebnisse macht diese ,greifbarer'. (c) Reallabor SRB 2018.

AP 5 (Wertschätzung durch Atmosphäre und Stimmung vermitteln): Insbesondere in der Arbeit mit marginalisierten Gruppen ist die Wortwahl wichtig, damit diese nicht eingeschüchtert werden, die Aufgabenstellung verstehen und das Gefühl haben, dass ihre Meinung wertgeschätzt wird. Im Projektteam sollte diskutiert werden, mit welcher Haltung diesen Gruppen begegnet werden soll, und in welchen 
Handlungen sich dies ausdrückt. Zudem ist es wichtig, dass die Sitzordnung im Sitzkreis, um einen kleinen Tisch herum oder an Stehtischen, dazu beiträgt, Hierarchien zwischen den Akteuren abzubauen.

AP 6 (Dokumentieren und Auswerten): In der Ideenwerkstatt (oder einem vergleichbaren Format) können Videoaufnahmen und Fotos die Arbeit dokumentieren. Produkte wie die Papphocker-Wand mit Handlungsempfehlungen der Akteure fassen die Ergebnisse der Diskussion zusammen und ermöglichen im Reflexionsworkshop eine unmittelbare Nutzung der Empfehlungen. Für die weitere Forschungsarbeit sind jedoch ergänzend umfangreiche Protokolle erforderlich, die z. B. gestützt auf Tonaufnahmen der Diskussionen und Präsentationen im Plenum erstellt werden können.

\subsection{Veranstaltung 5: Ziel-Beschreiterin}

Am Ende des Projektverlaufs, in der Abschlussphase, setzt Veranstaltung 5 ein. Zentral ist die Vorstellung der Ergebnisse aus dem Projekt mit Blick auf die Diffusion sowie die Implementierung und Etablierung (Verstetigung) der Ergebnisse. Ziel der letzten Veranstaltung ist zudem ein Rückblick auf das Projekt. Im Mittelpunkt steht die Integration der Ergebnisse in die Praxis und gleichzeitig die Verabschiedung des Projekts als Akteur aus dem Feld. Wesentlich ist auch die Danksagung an die externen Akteure sowie ein gemeinsamer feierlicher Abschluss.

\subsubsection{Steckbrief Ziel-Beschreiterin}

Wissensintegration und Bildungsziele: In dieser Veranstaltung wird vor allem Transformationswissen , aktiviert' und erzeugt. Dieses wiederum kann in die Praxis, in folgende und/oder in andere Projekte integriert werden.

Ablauf und Rollen: Vertreter(innen) des Projektteams stellen die Ergebnisse des Projekts als Ganzes vor. Die gezeigten Einblicke und Erkenntnisse werden in zwei Diskussionsrunden mit unterschiedlichen Schwerpunkten diskutiert. Alle ziehen zum Abschluss Resümee. Rolle der verschiedenen Akteure:

- Moderation: Projektteam, wobei die Praxispartner eine besondere Rolle spielen sollten

- Impulsreferate: Vertreter(innen) des Projektteams

- Teilnehmer(innen): Sind hier in erster Linie als Multiplikator(inn)en angesprochen 
Nutzen für externe Praxisakteure:

- Pflege ihres Netzwerks, Austausch mit Peers

- Einblicke in und Impulse aus der Forschung

- Impulse für das eigene Handeln in der Praxis

- Anregungen, wie sie auch außerhalb des Projekts Transformationen anregen und Transformationsprozesse gestalten können

Fragen, die die Inhalte der Ziel-Beschreiterin definieren: Was sind die Ergebnisse der Forschung? Was waren die Chancen und Herausforderungen und was wurde daraus gelernt? Wie geht es weiter?

Voraussetzungen, die für den Einsatz der Ziel-Beschreiterin erfüllt sein müssen:

- Gesamtergebnisse aus dem Projekt liegen vor.

- Erste Produkte (bspw. Publikationen), die sich an die Praxis richten, liegen möglichst vor oder können jedenfalls in Aussicht gestellt werden.

\subsubsection{Planungsaufgaben Ziel-Beschreiterin}

AP 1 (Identifizieren und Erreichen der Akteure): Zu dieser Veranstaltung werden alle eingeladen, die an einer der vorangegangenen Veranstaltungen teilgenommen haben. Zusätzlich sind gezielt weitere Akteure einzuladen, die mit Blick auf die Umsetzung eine besondere Rolle spielen könnten. Bei dieser Veranstaltung ist es überaus wichtig, dass die Praxispartner des Projekts prominent auftreten (z. B. für Präsentation, Einführung in eine Ausstellung, Moderation). Deren Teilnahme sollte bereits beim Anbahnen der Kooperation sichergestellt werden.

AP 2 (Inhaltliche Impulse aus dem Projekt bestimmen): Mit Blick auf die Darstellung der Projektergebnisse gilt es in der Projektplanung, die Synthesearbeiten so zu planen, dass deren Ergebnisse vorliegen. Im Mittelpunkt der Veranstaltung steht, nebst einer umfassenden Präsentation und Diskussion der Gesamtergebnisse des Projekts, die Reflexion der Zusammenarbeit zwischen Forscher(inne)n, Praxispartnern und externen Praxisakteuren: Was hat sich als besonders schwierig herausgestellt? Was hat sich bewährt, was sind unsere Lessons Learnt? Es empfiehlt sich, dafür eine Präsentation der Erfahrungen der Praxispartner in Form eines Vortrages mit anschließender Diskussionsrunde vorzusehen, in die externe Praxisakteure eingebunden werden.

AP 3 (Vorbereitungen speziell für externe Akteure treffen): Es bewährt sich, Broschüren mit einer Zusammenfassung der Ergebnisse und des Prozesses zu erstellen, welche die Teilnehmer(innen) mitnehmen können. 
AP 4 (Beteiligungsmethodik definieren): Die Projektergebnisse können auf vielfältige Art präsentiert werden (Poster, Vorträge, Ausstellung etc.), angesichts des in aller Regel beträchtlichen Umfangs empfiehlt sich die Wahl einer ,komprimierenden' Methode. Pecha Kucha (eine Vortragstechnik, in der 20 Folien jeweils 20 Sekunden lang eingeblendet werden) zum Beispiel ermöglicht es, in einer prägnanten Präsentation das Wesentliche der Forschungsergebnisse auf den Punkt zu bringen und auf anschauliche Weise einen Einblick in den Projektverlauf sowie die Forschungsergebnisse zu geben (zu Pecha Kucha s. z. B. https://www. pechakucha.com, zugegriffen am 22.04.2019). Eine anschließende Diskussion kann z. B. als Podiumsdiskussion gestaltet werden (s. hierzu Friesen et al. 2006), die als eine Art Interview realisiert wird. Dies bietet auch den Praxispartnern, die am Forschungsprozess beteiligt waren, die Möglichkeit, sich aktiv an der Gestaltung des Symposiums zu beteiligen (s. SRB-Box 5). Die Inhalte der so gestalteten Diskussionsrunde regen die Teilnehmer(innen) wiederum an, mit den Referent(inn)en und dem Projektteam in Dialog zu treten. Für das Forschungsprojekt wiederum bietet es die Chance, dass nicht nur kritisch nachgedacht, sondern ein Prozess zur Umsetzung und zur Formulierung weiterer Forschungsfragen angeregt wird.

\section{SRB-Box 5: Abschluss des Reallabors SRB: Resümee mit Praxispartnern in einem Inter- view}

Das Abschluss-Symposium stand am Ende der dreijährigen Förderzeit des Reallabors SRB. Das Ziel war es, Erfahrungen aus dem Projektverlauf zu teilen und Einblicke in die Ergebnisse zu geben. Zwei Schulleitungen, die als Praxispartner aktiv im Forschungsprojekt mitgearbeitet hatten, reflektierten mit Forscher(inne)n des Reallabors SRB die gemeinsame, kooperative Arbeit und ihre Lessons Learnt. Das Projektteam wollte so darstellen, wie die Kooperation im Projekt ablief und welchen direkten Nutzen die Partner im Projekt hatten. Im ersten Teil der anschließenden Diskussionsrunde wurden die Podiumsgäste dazu interviewt (s. Abb. 10) und berichteten von ihren sehr persönlichen Erfahrungen, aufgrund von Fragen wie: Was funktionierte gut? Wie aufwendig war das Ganze? Was kam dabei heraus? Was hätten wir anders machen können? Auf diese Art Feedback zu erhalten, war für die Forscher(innen) wertvoll. Auch für die externen Praxisakteure, die am Symposium teilnahmen, war es interessant, Kenntnis von der Wahrnehmung und den Erfahrungen der Praxispartner, bezogen auf die Zusammenarbeit im Reallabor, zu bekommen. Im zweiten Teil der Podiumsdiskussion wurde die Diskussionsrunde für Fragen und Anmerkungen aus dem Publikum geöffnet. Da im Publikum viele externe Akteure saßen, die bereits an vorangegangenen Symposien teilgenommen hatten, entstand dank des bereits aufgebauten Vertrauensverhältnisses eine anregende und inhaltsreiche Diskussion zwischen den externen Akteuren und den Praxispartnern und Forscher(innen) auf dem Podium. 

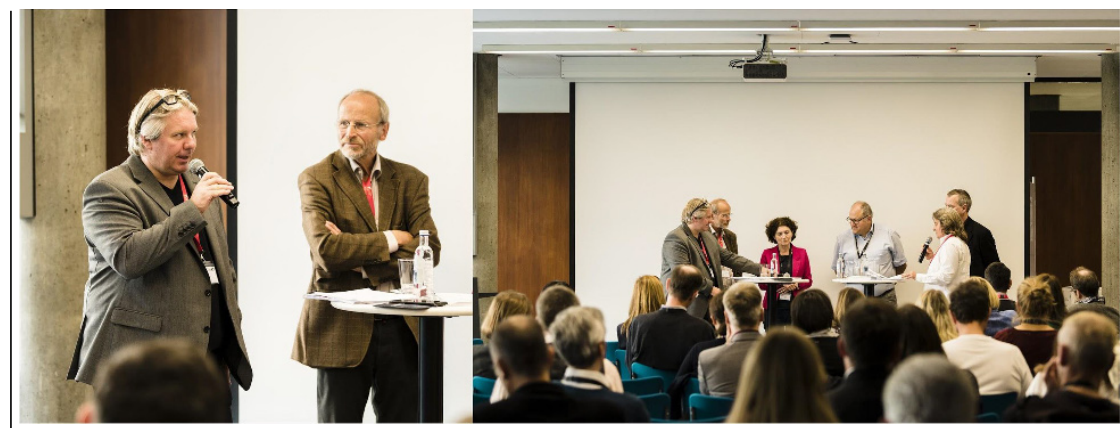

Abbildung 10: Forscher(innen) und Praxispartner bei der Podiumsdiskussion auf der Veranstaltung "Symposium STADT-RAUM-BILDUNG“". Podiumsdiskussionen können gehaltvoll sein, wenn sie sorgfältig vorbereitet und moderiert werden. (C) Reallabor SRB 2018.

AP 5 (Wertschätzung durch Atmosphäre und Stimmung vermitteln): In der Abschlussveranstaltung ist es wichtig, eine besondere Stimmung herzustellen und Wertschätzung auszudrücken. Es ist zu empfehlen, ein rückblickendes Element mit einzubauen, das auch emotionale Anknüpfungspunkte bietet und Erinnerungen wachruft an das gemeinsam Erlebte, z. B. einen Videozusammenschnitt der vorangegangenen Symposien (und weiterer Aktionen während der Forschung). Dazu können Fotos, Video- und Audioausschnitte benutzt werden, welche die Highlights des Forschungsprojekts zeigen und den Forschungsprozess zusammenfassen. Das dient nicht nur als kurzer Rückblick der vergangenen Jahre, sondern auch zur Einstimmung auf die Verabschiedung, indem die Zusammenarbeit mit den Praxispartnern und mit den externen Akteuren wertschätzend dargestellt wird. Eine Danksagung an alle Beteiligten schließt den offiziellen Teil ab. Um das Symposium ausklingen zu lassen, werden alle Anwesenden zum Umtrunk bei Musik und Häppchen eingeladen, so dass die gemeinsame Arbeit abschließend gefeiert und das Forschungsprojekt als Akteur verabschiedet werden kann.

AP 6 (Dokumentieren und Auswerten): Auch das Abschlusssymposium sollte durch Videoaufnahmen und Fotos dokumentiert werden, wobei die Tonspur der Videoaufzeichnung transkribiert und ausgewertet werden kann. Alternativ können die Interviewergebnisse protokolliert werden, womit der Transkriptionsaufwand entfällt. Im Reflexionsworkshop wird nochmals Resümee gezogen; vor allem wird an den Lessons Learnt gearbeitet. 


\subsection{Reflexionsworkshop jeweils am Tag nach dem Symposium}

Reflexion und Aufbereitung der Impulse aus den Symposien: Die Impulse der externen Akteure aus den Symposien werden im internen Reflexionsworkshop, der jeweils am nächsten Tag stattfindet, reflektiert und aufgearbeitet. Dies ersetzt natürlich nicht eine weitergehende sorgfältige Ergebnisaufbereitung, dient aber dazu, die noch frischen Eindrücke zu vergemeinschaften und die nächsten Schritte zu planen. Gestützt auf ihre Notizen und auf die Produkte aus dem jeweiligen Symposium sammeln und clustern alle Teammitglieder die aus ihrer Sicht relevanten Ergebnisse, Einschätzungen und Fragen. Anschließend diskutieren sie diese in teilprojektübergreifenden Kleingruppen von drei bis fünf Personen mit Blick auf die Konsequenzen für das Projekt als Ganzes und seine Teile, bevor sich dann die einzelnen Teams der Teilprojekte zusammensetzen und über die Integration der Ergebnisse in das jeweilige Teilprojekt beraten. Gemeinsam wird abschließend ein Arbeitsplan erstellt, in dem festgehalten wird, wie die weitere Auswertung der Symposiumsergebnisse erfolgt und wie diese im Projekt umgesetzt werden sollen. Damit dies möglich ist, gilt es, bei der Projektplanung entsprechende Flexibilität im Design vorzusehen.

Inhaltliche Vorbereitung mit Blick auf die interne Vernetzung: Damit der Reflexionsworkshop seine Funktion im Syntheseprozess erfüllen kann, ist über die Vorbereitung auf das jeweilige Symposium hinaus eine Aufbereitung des Arbeitsstands in den Teilprojekten für interne Zwecke erforderlich. Dazu legen alle Teilprojekte ihren jeweils aktuellen Zwischenstand sowie offene Fragen und Herausforderungen dar. Um die Arbeitslast insgesamt in Grenzen zu halten, empfiehlt es sich, dies anhand eines Handouts mit vorgegebener Gliederung zu tun: Ziel und Fragestellung, Arbeitsstand bis dato, erste wesentliche Erkenntnisse, Schwierigkeiten, anstehende Entscheidungen und (neue) Schnittstellen zu anderen Teilprojekten, geplante Arbeitsschritte. Ein solches Handout kann zu Beginn der Arbeit erstellt werden, dann für jeden Reflexionsworkshop von den Teilprojekten auf den aktuellen Stand gebracht und zur Vorbereitung des Workshops vorher von der Projektkoordination an alle Teammitglieder geschickt werden. 


\section{7 Übersicht über die Veranstaltungen der Exchange- Veranstaltungsreihe}

Tab. 2 gibt eine Übersicht zu allen fünf Veranstaltungen der Exchange-Veranstaltungsreihe und stellt die Schwerpunkte je Veranstaltung mithilfe von Fragen dar: Sie erläutert,

- wann die Veranstaltung im Verlauf des Projekts sinnvoll einzusetzen ist;

- wie weit das Projekt jeweils fortgeschritten sein muss, um inhaltlich wie auch strukturell Beiträge in der Veranstaltung leisten zu können;

- die möglichen Inhalte der Veranstaltung;

- das Ziel und den Nutzen der Veranstaltung für das Projekt;

- was Praxisakteure, die nicht in das Projekt eingebunden sind, von der Veranstaltung haben. 
Tabelle 2: Schwerpunkte der fünf Veranstaltungen der Exchange-Veranstaltungsreihe.

\begin{tabular}{|c|c|c|}
\hline & $\begin{array}{l}\text { Veranstaltung } 1 \\
\text { Tür-Öffnerin }\end{array}$ & $\begin{array}{l}\text { Veranstaltung } 2 \\
\text { Weg-Bereiterin (optional) }\end{array}$ \\
\hline $\begin{array}{l}\text { Wann im Projekt- } \\
\text { verlauf sollte die } \\
\text { Veranstaltung } \\
\text { eingesetzt wer- } \\
\text { den? }\end{array}$ & $\begin{array}{l}\text { Einstiegsphase } \\
\text { 3-jähriges Projekt, ohne/mit 2-stu- } \\
\text { figem Antragsverfahren: 9. Monat / } \\
\text { 4. Monat } \\
\text { 5-jähriges Projekt, ohne/mit 2-stu- } \\
\text { figem Antragsverfahren: } 12 \text {. Monat / } \\
\text { 7. Monat }\end{array}$ & $\begin{array}{l}\text { Orientierungsphase } \\
\text { 3-jähriges Projekt, ohne/mit 2-stufi- } \\
\text { gem Antragsverfahren: - / optional } \\
\text { 13. Monat } \\
\text { 5-jähriges Projekt, ohne/mit 2-stufi- } \\
\text { gem Antragsverfahren: } 21 \text {. Monat / } \\
\text { 16. Monat }\end{array}$ \\
\hline $\begin{array}{l}\text { Was setzt die } \\
\text { Veranstaltung } \\
\text { voraus, mit Blick } \\
\text { auf das, was im } \\
\text { Projekt bereits } \\
\text { erreicht wurde? }\end{array}$ & $\begin{array}{l}\text { - Projektteam steht fest. } \\
\text { - Forschungsthemen und Fragestel- } \\
\text { lungen sind definiert. } \\
\text { - Relevante Akteure im Feld (Praxis } \\
\text { und Forschung) sind identifiziert. }\end{array}$ & $\begin{array}{l}\text { - Falls mit Fallbeispielen gearbeitet } \\
\text { wird: Fallbeispiele für die For- } \\
\text { schung sind ausgewählt. } \\
\text { - Erste Forschungserfahrungen, } \\
\text { erste Transformationserfahrungen } \\
\text { liegen vor. }\end{array}$ \\
\hline $\begin{array}{l}\text { Welche Fragen } \\
\text { definieren die } \\
\text { Inhalte der Veran- } \\
\text { staltung? }\end{array}$ & $\begin{array}{l}\text { Was sind Forschungsthema und For- } \\
\text { schungsvorhaben? Wer ist das Pro- } \\
\text { jektteam? Welche Expertise bringt } \\
\text { es mit? Welche Rolle wird es im Feld } \\
\text { einnehmen? Wie reagieren die ex- } \\
\text { ternen Akteure auf das Projekt? }\end{array}$ & $\begin{array}{l}\text { Wie wird geforscht? Wie soll der For- } \\
\text { schungsprozess aussehen? Welche } \\
\text { Methoden sollen eingesetzt werden } \\
\text { (ggf.: Welche Fallbeispiele bilden den } \\
\text { Untersuchungsgegenstand?) und } \\
\text { sind diese geeignet? }\end{array}$ \\
\hline $\begin{array}{l}\text { Was sind das Ziel } \\
\text { und der Nutzen } \\
\text { der Veranstaltung } \\
\text { für das Projekt? }\end{array}$ & $\begin{array}{l}\text { - Etablierung Forschungsprojekt } \\
\text { - Gewinnung von (weiteren) Praxis- } \\
\text { akteuren für die transdisziplinäre } \\
\text { Zusammenarbeit } \\
\text { - Vorstellung des Forschungspro- } \\
\text { jekts und des Projektteams } \\
\text { - Das im Forschungsantrag darge- } \\
\text { legte Zielwissen wird durch exter- } \\
\text { ne Akteure reflektiert und ergänzt }\end{array}$ & $\begin{array}{l}\text { - Transparente Darstellung des For- } \\
\text { schungsgegenstands, Berichte der } \\
\text { Praxispartner (z. B. aus Fallbeispie- } \\
\text { len des Projekts) } \\
\text { - Die Integration von Praxiswissen in } \\
\text { den Forschungsprozess }\end{array}$ \\
\hline $\begin{array}{l}\text { Worin liegt der } \\
\text { Nutzen für Praxis- } \\
\text { akteure, die nicht } \\
\text { in das Projekt } \\
\text { eingebunden sind? }\end{array}$ & $\begin{array}{l}\text { - Erweiterung und Pflege ihres } \\
\text { Netzwerks, Austausch mit Peers } \\
\text { - Horizonterweiterung, Erwerb } \\
\text { neuen Wissens }\end{array}$ & $\begin{array}{l}\text { - Pflege ihres Netzwerks, Austausch } \\
\text { mit Peers } \\
\text { - Einblicke in die Perspektive und } \\
\text { Erfahrungen Dritter, daraus Im- } \\
\text { pulse für die eigene Arbeit }\end{array}$ \\
\hline
\end{tabular}


Forts. Tabelle 2

\begin{tabular}{|c|c|c|}
\hline $\begin{array}{l}\text { Veranstaltung } 3 \\
\text { Weg-Begleiterin }\end{array}$ & $\begin{array}{l}\text { Veranstaltung } 4 \\
\text { Ziel-Erspäherin (optional) }\end{array}$ & $\begin{array}{l}\text { Veranstaltung } 5 \\
\text { Ziel-Beschreiterin }\end{array}$ \\
\hline Arbeitsphase & Arbeitsphase & Abschlussphase \\
\hline $\begin{array}{l}\text { 3-jähriges Projekt, ohne/mit } \\
\text { 2-stufigem Antragsverfahren: } \\
\text { 18. / 13. oder 22. Monat }\end{array}$ & $\begin{array}{l}\text { 3-jähriges Projekt, ohne/mit } \\
\text { 2-stufigem Antragsverfahren: } \\
\text { - / optional 22. Monat }\end{array}$ & $\begin{array}{l}\text { 3-jähriges Projekt, ohne/mit } \\
\text { 2-stufigem Antragsverfahren: } \\
\text { 27. / 31. Monat }\end{array}$ \\
\hline $\begin{array}{l}\text { 5-jähriges Projekt, ohne/mit } \\
\text { 2-stufigem Antragsverfahren: } \\
\text { 30. / 25. Monat }\end{array}$ & $\begin{array}{l}\text { 5-jähriges Projekt, ohne/mit } \\
\text { 2-stufigem Antragsverfahren: } \\
\text { 39. / 34. und/oder 43. Monat }\end{array}$ & $\begin{array}{l}\text { 5-jähriges Projekt, ohne/mit } \\
\text { 2-stufigem Antragsverfahren: } \\
\text { 48. / 52. Monat }\end{array}$ \\
\hline $\begin{array}{l}\text { - Erste Erkenntnisse und Er- } \\
\text { fahrungen aus dem Projekt } \\
\text { liegen vor. } \\
\text { - Für das Projekt besonders } \\
\text { spannende Forschungsfel- } \\
\text { der und -projekte, von de- } \\
\text { nen gelernt werden kann, } \\
\text { sind bekannt. }\end{array}$ & $\begin{array}{l}\text { - Wesentliche Teilergebnisse } \\
\text { aus dem Projekt liegen vor. } \\
\text { - Überblick über Akteure im } \\
\text { Feld, die bisher außen vor } \\
\text { blieben, mit Blick auf die Pra- } \\
\text { xisrelevanz und Legitimität } \\
\text { der Ergebnisse aber beteiligt } \\
\text { werden sollten, besteht. }\end{array}$ & $\begin{array}{l}\text { - Gesamtergebnisse aus dem } \\
\text { Projekt liegen vor. } \\
\text { - Erste Produkte, die sich an } \\
\text { die Praxis richten, liegen } \\
\text { möglichst vor oder können } \\
\text { jedenfalls in Aussicht ge- } \\
\text { stellt werden. }\end{array}$ \\
\hline $\begin{array}{l}\text { Was sind erste Erkenntnisse } \\
\text { und Erfahrungen, die das } \\
\text { Projektteam gesammelt hat? } \\
\text { Gibt es weitere Forschungs- } \\
\text { projekte oder Initiativen im } \\
\text { selben Themenbereich? }\end{array}$ & $\begin{array}{l}\text { Was sind die ersten Zwischen- } \\
\text { ergebnisse der Forschung? } \\
\text { Welche Erkenntnisse wurden } \\
\text { bereits gewonnen? Wie werden } \\
\text { bereits erhobene Daten bis zum } \\
\text { Ende ausgewertet? }\end{array}$ & $\begin{array}{l}\text { Was sind die Ergebnisse der } \\
\text { Forschung? Was waren die } \\
\text { Chancen und Herausforderun- } \\
\text { gen und was wurde daraus } \\
\text { gelernt? Wie geht es weiter? }\end{array}$ \\
\hline $\begin{array}{l}\text { - Erste Diskussion der prak- } \\
\text { tischen Anwendbarkeit } \\
\text { erster Eindrücke aus der } \\
\text { Forschung } \\
\text { - Rückkopplung der Erfah- } \\
\text { rungen mit dem wissen- } \\
\text { schaftlichen Diskurs }\end{array}$ & $\begin{array}{l}\text { - Vorstellung erster Teilergeb- } \\
\text { nisse } \\
\text { - Rückspiegelung der Erkennt- } \\
\text { nisse aus der Forschung an } \\
\text { die externen Praxisakteure } \\
\text { - Gemeinsame Erarbeitung von } \\
\text { Transformationswissen }\end{array}$ & $\begin{array}{l}\text { - Darstellung der Gesamter- } \\
\text { gebnisse und deren Umset- } \\
\text { zung } \\
\text { - Generiertes Transforma- } \\
\text { tionswissen kann in die Pra- } \\
\text { xis und in folgende / andere } \\
\text { Projekte integriert werden }\end{array}$ \\
\hline $\begin{array}{l}\text { - Pflege ihres Netzwerks, } \\
\text { Austausch mit Peers } \\
\text { - Impulse aus der Forschung } \\
\text { und Einblicke in die For- } \\
\text { schung inner- und außer- } \\
\text { halb des Projekts } \\
\text { - Impulse für das praktische } \\
\text { Handeln in den eigenen } \\
\text { Problemstellungen }\end{array}$ & $\begin{array}{l}\text { - Pflege ihres Netzwerks, Aus- } \\
\text { tausch mit Peers } \\
\text { - Einblicke in und Impulse aus } \\
\text { der Forschung } \\
\text { - Impulse für das praktische } \\
\text { Handeln mit Bezug auf ihre } \\
\text { Problemstellungen } \\
\text { - Empowerment und Hinweise, } \\
\text { wie sie auch nach Ende des } \\
\text { Projekts Transformationspro- } \\
\text { zesse gestalten können }\end{array}$ & $\begin{array}{l}\text { - Pflege ihres Netzwerks, Aus- } \\
\text { tausch mit Peers } \\
\text { - Einblicke in und Impulse aus } \\
\text { der Forschung } \\
\text { - Impulse für das eigene Han- } \\
\text { deln in der Praxis } \\
\text { - Anregungen, wie sie auch } \\
\text { außerhalb des Projekts } \\
\text { Transformationen anregen } \\
\text { und Transformationsprozes- } \\
\text { se gestalten können }\end{array}$ \\
\hline
\end{tabular}




\section{Reflexion}

Die Idee, eine Veranstaltungsreihe als Planungs- und Steuerungsinstrument für transdisziplinäre Forschungsprojekte einzusetzen, entstand auf Basis der Erfahrungen aus dem Reallabor SRB. In diesem wurden schon frühzeitig, während der Auftragsphase, mehrere Symposien mit anschließenden Reflexionsworkshops für die einzelnen Projektphasen geplant und in der Laufzeit umgesetzt. Der Veranstaltungsrhythmus lag bei durchschnittlich sechs Monaten. Bei einer Gesamtdauer des Projekts von drei Jahren war dies durchaus ambitioniert, und die teilweise hohe zeitliche Belastung der Forscher(innen), die die Veranstaltungen inhaltlich ausgestalten, auswerten und deren Ergebnisse umsetzen mussten, stellte oftmals eine große Herausforderung im Reallabor SRB dar.

Im Projektverlauf des Reallabors SRB hat sich die Einbindung von externen Akteuren als wichtiger Kern für die Arbeit herausgestellt. Die Veranstaltungsreihe ermöglichte eine konsistente und kontinuierliche Kommunikation mit Praxisakteuren, so dass ,Betroffene' und Interessierte im Forschungsprozess mitgenommen und sowohl an Entscheidungen über den Forschungsprozess als auch an Ergebnissen teilhaben konnten. Über die verschiedenen öffentlichen Symposien konnten bereits zu Beginn das Forschungsvorhaben, Erfahrungsberichte, Teilergebnisse und zuletzt die Gesamtergebnisse des Projekts mit einer breiteren Öffentlichkeit reflektiert werden, was von Beginn an ermöglichte, neues Wissen und Impulse für die Forschung einzuholen. Gerade für die Schulentwicklung, in der die politische Entscheidungsfindung großen Raum einnimmt, war eine transparente, öffentliche Darstellung, Diskussion und Bewertung des Forschungsprozesses mit externen Praxisakteuren und auch mit externen Wissenschaftler(inne)n erheblich für eine hohe Qualität des Prozesses.

Die Symposien boten den Forscher(inne)n die Möglichkeit, neue Kontakte zu externen Forscher(inne)n herzustellen und diese zu verstetigen. Die Praxisakteure wiederum knüpften Kontakte untereinander, so dass sie sich gegenseitig bei ihrer beruflichen Tätigkeit unterstützen konnten und dies auch weiterhin tun können. Die Symposien förderten somit die Entstehung eines Netzwerks sowohl für die Forscher(innen) als auch für die Praxisakteure, das die Laufzeit des Reallabors SRB überdauert hat.

Die internen Reflexionsworkshops boten den Forscher(inne)n im Projektteam die Möglichkeit, die Erkenntnisse aus dem jeweils vorangegangenen Symposium quer zu den beteiligten Disziplinen und Teilprojekten zu diskutieren, zu reflektieren und zu werten. Dies ermöglichte den Transfer von hoch aktuellem Praxiswissen in die laufende Forschung. Die regelmäßige Auf- und Nachbereitung der Ergebnisse aus den Symposien nahm zeitlich viele Kapazitäten in Anspruch, führte aber dazu, dass die Ergebnisse für Akteure aus der Forschung und der Praxis des Schulbaus anschlussfähiger wurden. Dieser Erfolg ist zu großen Teilen 
auf die kombinierte Reflexion durch die Symposien und die Reflexionsworkshops zurückzuführen. Neben der Funktion der Diffusion, des Austauschs und der Vernetzung wirkte die Veranstaltungsreihe zudem strukturgebend für das Reallabor SRB, indem sie die Meilensteine setzte, an denen eine Reflexion innerhalb des Projekts stattfinden musste.

Der hohe organisatorische Aufwand für die Veranstaltungsreihe in der Planung, Durchführung und Dokumentation hat sich gelohnt, weil das Ergebnis von allen (internen und externen) Akteuren insgesamt sehr positiv aufgenommen und sehr wertgeschätzt wurde und die produktive Zusammenarbeit zwischen Wissenschaftler(inne)n und Praxisakteuren erhöhte. Eine wichtige Rolle spielten dabei auch die Vermittlung von Wertschätzung gegenüber den Praxisakteuren und das Schaffen einer vertrauensvollen Atmosphäre in den Symposien. Diese beiden Aspekte - Wertschätzung vermitteln und Vertrauen schaffen - bilden eine wesentliche Grundlage für den erfolgreichen Einsatz der Exchange-Veranstaltungsreihe als Planungs- und Steuerungsinstrument.

\section{Dank}

Die Autor(inn)en danken Richard Beecroft für die Diskussionen über eine frühere Fassung des Textes. Insbesondere möchten sie Marius Albiez, Laura Gebhardt, Andri König und Thomas Potthast sowie zwei anonymen Gutachter(inne)n für die konkreten Hinweise zur Verbesserung des Textes im Rahmen des internen und externen Reviews danken. Schließlich danken die Autor(inn)en den beiden Herausgebenden, Rico Defila und Antonietta Di Giulio, für ihre Rückmeldungen zum Text. 


\section{Literatur}

Alcántara, S., Quint, A., \& Seebacher, A. (2018). Der Partizipationsmythos „Partizipation in Reallaboren muss repräsentativ sein". In R. Defila \& A. Di Giulio (Hrsg.), Transdisziplinär und transformativ forschen. Eine Methodensammlung (S. 137-159). Wiesbaden: Springer VS. doi: 10.1007/978-3-658-21530-9_7.

Arnold, A., \& Piontek, F. M. (2018). Zentrale Begriffe im Kontext der Reallaborforschung. In R. Defila \& A. Di Giulio (Hrsg.), Transdisziplinär und transformativ forschen. Eine Methodensammlung (S. 143-154). Wiesbaden: Springer VS. doi: 10.1007/ 978-3-658-21530-9_8.

Beecroft, R., Trenks, H., Rhodius, R., Benighaus, C., \& Parodi, O. (2018). Reallabore als Rahmen transformativer und transdisziplinärer Forschung: Ziele und Designprinzipien. In R. Defila \& A. Di Giulio (Hrsg.), Transdisziplinär und transformativ forschen. Eine Methodensammlung (S. 75-100). Wiesbaden: Springer VS. doi: 10.1007/978-3-65821530-9_4.

Benighaus, C., \& Benighaus, L. (2016). Diskussion und Vorsorge von Hagelereignissen. In C. Benighaus, G. Wachinger \& O. Renn (Hrsg.), Bürgerbeteiligung - Konzepte und Lösungswege für die Praxis (S. 216-222). Berlin, Frankfurt a. M.: Wolfgang Metzner Verlag.

Benighaus, C., \& Renn, O. (2016). Teil A Grundlagen. In C. Benighaus, G. Wachinger \& O. Renn (Hrsg.), Bürgerbeteiligung - Konzepte und Lösungswege für die Praxis (S. 17102). Berlin, Frankfurt a. M.: Wolfgang Metzner Verlag.

Bergold, J., \& Thomas, S. (2010). Partizipative Forschung. In G. Mey \& K. Mruck (Hrsg.), Handbuch Qualitative Forschung in der Psychologie (S. 333-344). Wiesbaden: Springer VS.

Brown, J., \& Isaacs, D. (2007). Das World Café: Kreative Zukunftsgestaltung in Organisationen und Gesellschaft. Heidelberg: Carl-Auer-Systeme Verlag.

Defila, R., Di Giulio, A., \& Scheuermann, M. (2006). Forschungsverbundmanagement: Handbuch für die Gestaltung inter- und transdisziplinärer Projekte. Zürich: vdf Hochschulverlag an der ETH Zürich.

Eckart, J., Ley, A., Häußler, E., \& Erl, Th. (2018). Leitfragen für die Gestaltung von Partizipationsprozessen in Reallaboren. In R. Defila \& A. Di Giulio (Hrsg.), Transdisziplinär und transformativ forschen. Eine Methodensammlung (S. 105-135). Wiesbaden: Springer VS. doi: 10.1007/978-3-658-21530-9_6.

Friesen, Kaye and Associates (2006). Tips for Facilitating Panel Discussions. https://www.fka.com/files/TIPS-Facilitating-Panel-Discussions.pdf. Zugegriffen am 18.01.2019.

Godemann, J. (2005). Verständigung als Basis inter- und transdisziplinärer Zusammenarbeit. In G. Michelsen \& J. Godemann (Hrsg.), Handbuch der Nachhaltigkeitskommunikation. Grundlagen und Praxis (S. 85-95). München: oekom.

Hofmann, S. (Hrsg.) (2014). Partizipation macht Architektur. Die Baupiloten-Methode und Projekte. Berlin: Jovis. 
Quint, A., Alcántara, S., \& Seebacher, A. (2018). Der Partizipationsmythos „Partizipation in Reallaboren ist per se transparent und muss es auch sein“. In R. Defila \& A. Di Giulio (Hrsg.), Transdisziplinär und transformativ forschen. Eine Methodensammlung (S. 69-73). Wiesbaden: Springer VS. doi: 10.1007/978-3-658-21530-9_3.

Renn, O., \& Benighaus, C. (2003). Diskurs: Vorgehen, Bedingungen, Klassifikation und Chancen. In A. Ley \& L. Weitz (Hrsg.), Praxis Bürgerbeteiligung: Ein Methodenhandbuch (Arbeitshilfen für Selbsthilfe- und Bürgerinitiativen, Nr. 30) (S. 108-114). Bonn: Stiftung Mitarbeit.

Rogge, K. I. (2000). Ideenwerkstatt. In H.-W. Kuhn \& P. Massing (Hrsg.), Methoden und Arbeitstechniken (Lexikon der politischen Bildung, Bd. 3) (S. 77-78). Schwalbach am Taunus: Wochenschau Verlag.

Schäpke, N., Stelzer, F., Bergmann, M., Singer-Brodowski, M., Wanner, M., Caniglia G., \& Lang, D. (2017). Reallabore im Kontext transformativer Forschung. Ansatzpunkte zur Konzeption und Einbettung in den internationalen Forschungsstand. (No. 1/2017). Leuphana Universität Lüneburg, Institut für Ethik und Transdisziplinäre Nachhaltigkeitsforschung. http://hdl.handle.net/10419/168596. Zugegriffen am 20.05.2019.

Seebacher, A., Alcántara, S., \& Quint, A. (2018). Akteure in Reallaboren - Reallabore als Akteure. In R. Defila \& A. Di Giulio (Hrsg.), Transdisziplinär und transformativ forschen. Eine Methodensammlung (S. 155-159). Wiesbaden: Springer VS. doi: 10.1007/ 978-3-658-21530-9_9.

United Nations Human Rights, Office of the Commissioner (2012). Fishbowl: The art of active listening. http://slitoolkit.ohchr.org/data/downloads/fishbowl.pdf. Zugegriffen am 18.01.2019.

Open Access Dieses Kapitel wird unter der Creative Commons Namensnennung 4.0 International Lizenz (http://creativecommons.org/licenses/by/4.0/deed.de) veröffentlicht, welche die Nutzung, Vervielfältigung, Bearbeitung, Verbreitung und Wiedergabe in jeglichem Medium und Format erlaubt, sofern Sie den/die ursprünglichen Autor(en) und die Quelle ordnungsgemäß nennen, einen Link zur Creative Commons Lizenz beifügen und angeben, ob Änderungen vorgenommen wurden.

Die in diesem Kapitel enthaltenen Bilder und sonstiges Drittmaterial unterliegen ebenfalls der genannten Creative Commons Lizenz, sofern sich aus der Abbildungslegende nichts anderes ergibt. Sofern das betreffende Material nicht unter der genannten Creative Commons Lizenz steht und die betreffende Handlung nicht nach gesetzlichen Vorschriften erlaubt ist, ist für die oben aufgeführten Weiterverwendungen des Materials die Einwilligung des jeweiligen Rechteinhabers einzuholen.

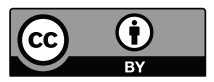

